\title{
Soft-Collinear Factorization in Effective Field Theory
}

\author{
Christian W. Bauer, Dan Pirjol, and Iain W. Stewart \\ Department of Physics, University of California at San Diego, \\ 9500 Gilman Drive, La Jolla, CA 92093-0319, USA
}

\begin{abstract}
The factorization of soft and ultrasoft gluons from collinear particles is shown at the level of operators in an effective field theory. Exclusive hadronic factorization and inclusive partonic factorization follow as special cases. The leading order Lagrangian is derived using power counting and gauge invariance in the effective theory. Several species of gluons are required, and softer gluons appear as background fields to gluons with harder momenta. Two examples are given: the factorization of soft gluons in $B \rightarrow D \pi$, and the soft-collinear convolution for the $B \rightarrow X_{s} \gamma$ spectrum.
\end{abstract}




\section{INTRODUCTION}

Many processes that can be examined at current and future colliders involve hadrons with energy much larger than their mass. For such processes the large energy $Q \gg \Lambda_{\mathrm{QCD}}$ defines a scale that can be described by perturbative QCD. It is convenient to use light cone coordinates $p^{\mu}=\left(p^{+}, p^{-}, p^{\perp}\right)$, where $p^{+}=n \cdot p$ and $p^{-}=\bar{n} \cdot p$, and the light cone unit vectors satisfy $n^{2}=\bar{n}^{2}=0$ and $n \cdot \bar{n}=2$. For an energetic hadron the relevant momentum scales are the large $\bar{n} \cdot p$ component $Q$, the transverse momentum $p_{\perp}$, and the $n \cdot p$ component of order $p_{\perp}^{2} / Q$. The dynamics of these hadrons can be described in a systematic way by constructing a soft-collinear effective theory (SCET) [1 3]. Fluctuations with momenta $p^{2} \gtrsim Q^{2}$ are integrated out and appear in Wilson coefficients, while fluctuations with momenta $p^{2} \ll Q^{2}$ appear in time ordered products of effective theory fields. The effective theory is organized as an expansion in powers of a small parameter $\lambda$, where in typical processes either $\lambda=\Lambda_{\mathrm{QCD}} / Q$ or $\lambda=\sqrt{\Lambda_{\mathrm{QCD}} / Q}$.

Traditionally, energetic processes in QCD are described with the help of factorization theorems, which separate the different scales from one another [4,5]. In general, for inclusive processes the leading twist cross section is a convolution of a hard scattering kernel $H$, a jet function $J$, and a soft function $S$,

$$
\sigma \sim H \otimes J \otimes S
$$

The function $H$ encodes the short distance physics, the jet function describes the propagation of energetic particles in collimated jets, and the soft function contains nonperturbative long distance physics. Examples of processes which can be described with Eq. (百) include DrellYan and large $x$ deep inelastic scattering [5], as well as inclusive $B$ decays in certain regions of phase space [6 9]. A similar type of factorization also occurs for exclusive processes such as $e^{-} \gamma \rightarrow e^{-} \pi, \pi^{+} p \rightarrow \pi^{+} p, \gamma^{*} \gamma^{*} \rightarrow \pi \pi$ [10,11], and decays involving heavy quarks such as $B \rightarrow D \pi$ [12 14]. Here cross sections or decay rates are written as convolutions of hard scattering kernels $T$, light-cone hadron wave functions $\Phi$, and soft form factors $F$,

$$
\Gamma \sim T \otimes \Phi \otimes F
$$

where the hard scattering kernels describe the short distance part of the process, the lightcone wave functions describe the collinear dynamics of the energetic mesons, and the form factors encode soft interactions of the hadrons. To prove factorization formulae, momentum regions that give rise to infrared divergences via pinch surfaces are identified with the help of the Coleman-Norton theorem and Landau equations [5]. These pinch surfaces are reproduced by reduced graphs where all off-shell lines are shrunk to a point. Finally, the set of reduced graphs that contribute at leading power are obtained by a power counting for the strength of the infrared divergence. Despite its many strengths, the fact that the power counting occurs only at the end is a complication in this approach. An alternative is to identify infrared divergences using the method of regions or threshold expansion 15. In this case the complete expansion of a diagram in QCD is reproduced by adding diagrams that are homogeneous in 
the expansion parameter. These diagrams are defined by a particular scaling for their loop momenta. The main advantages of this approach are in fixed order computations. In this case complications occur due to the fact that many momentum regions, both onshell and offshell, must be considered. In many ways the power of the effective theory we discuss is that it synthesizes the advantages of these two approaches.

There are several reasons why an effective theory for energetic processes may simplify their description. The basic idea is to focus solely on the physical infrared degrees of freedom in such a way that the power counting is present from the start. Symmetries which emerge in the large energy limit are then explicit in the effective action, power corrections are simply given by matrix elements of operators that are higher order in the power counting, and Sudakov double logarithms are summed by solving renormalization group equations in the effective theory. In this paper we present some details of the structure of the effective theory focusing on interactions involving the softer gluons. In particular we wish to explain how soft-collinear factorization can be addressed in a simple universal way in the SCET at leading order in the power counting.

Reproducing the infrared physics for interactions between energetic and non-energetic massless particles in QCD requires three classes of effective theory fields: collinear modes with $\left(p^{+}, p^{-}, p^{\perp}\right) \sim Q\left(\lambda^{2}, 1, \lambda\right)$, soft modes with $p^{\mu} \sim Q \lambda$, and ultrasoft (usoft) modes' with $p^{\mu} \sim Q \lambda^{2}$. Fluctuations dominated by other regions of momenta are integrated out. For heavy quarks the large mass is factored out as in the Heavy Quark Effective Theory (HQET) [16]. Depending on the value of $\lambda$, heavy quarks with residual momentum $\sim \Lambda_{\mathrm{QCD}}$ are then included in either the soft or usoft category.

Since the SCET involves more than one distinct gluon field the nature of gauge symmetry is richer than in full QCD. In particular it is necessary to use the idea of background fields [17] to give well defined meaning to several distinct gluon fields. Technically, this occurs due to the necessity of defining each gluon as the gauge particle associated with a local non-Abelian symmetry. However, physically the necessity of a background field treatment is evident. For instance, usoft gluons with $p^{2} \simeq\left(Q \lambda^{2}\right)^{2}$ fluctuate over much larger distance scales than collinear fields with offshellness $p^{2} \simeq(Q \lambda)^{2}$. Thus, the picture is simply that usoft gluons produce a smooth non-Abelian background through which collinear particles propagate. For Drell-Yan, it was noted long ago by Tucci [18] that the proof of usoft-collinear factorization is simplified by using background field Feynman gauge. In constructing the SCET certain background fields are a necessity, not simply a tool. We will see that background fields also play a role in understanding factorization and deriving Feynman rules for soft gluons.

At lowest order in the SCET only $n \cdot A_{s}$ soft and $n \cdot A_{u s}$ usoft gluons interact with collinear quarks [19] and collinear gluons [14]. Furthermore, a consistent power counting in $\lambda$ requires

\footnotetext{
${ }^{1}$ Often for a specific physical process only soft or usoft gluons are relevant, and the generic term "soft" may be adopted. For the sake of generality we will describe soft and usoft particles separately.
} 
that the interaction of $(\mathrm{u})$ soft gluons with collinear fields are multipole expanded [1, 2]. 2] As a result only the $n \cdot p$ momenta of a collinear particle can be changed by interaction with an usoft gluon. We will show that these facts lead to simplifications in the structure of SCET matrix elements, traditionally referred to as the factorization of usoft gluons from collinear jets [21]. A transformation is given for the collinear fields which decouples all usoft gluons from the collinear Lagrangian, at the expense of complicating the form of the operators responsible for production and decay. Similar results are found for soft gluons once offshell fluctuations with momenta $p^{\mu} \sim Q(\lambda, 1, \lambda)$ are integrated out. The benefit of the effective theory is that the above results are very general, and can be applied in a universal way to many processes. Since the coupling of all (u)soft particles appear only in the operators responsible for the process, cancellations and simplifications that appear at leading order in $\lambda$ are easier to see.

We begin in section $\llbracket$ by discussing HQET in the language of Wilson lines. This allows us to introduce in a simple context some of the techniques we will need for (u)soft gluons. In section [II we explain how the leading (u)soft and collinear Lagrangians follow from invariance under gauge symmetries in the effective theory. In section IV] we discuss how usoft and soft gluons factor from collinear particles in the effective theory. In section $\square$ we give two examples of the effective theory formalism, followed by conclusions in section $\mathrm{V} 1$. In Appendix $\mathrm{A}$ we show how gauge invariant soft-collinear operators are obtained by integrating out offshell fluctuations.

In section $\nabla \mathrm{A}$ we take as an example the decays $B^{-} \rightarrow D^{0} \pi^{-}$and $B^{0} \rightarrow D^{+} \pi^{-}$at leading order in $\lambda=\Lambda_{\mathrm{QCD}} / Q$. These exclusive decays are mediated by four-quark operators for which a generalized factorization formula was proposed in Refs. [12,13]. In this example soft interactions factor but do not cancel. Also a nontrivial convolution occurs between the hard Wilson coefficient and the collinear degrees of freedom which give the light-cone pion wavefunction. In Ref. 22 the generalized factorization formula was shown to be valid at two-loop order. In Ref. [14 a proof of this formula was given to all orders in perturbation theory. In this section we give a more detailed explanation of the part of the proof involving the decoupling of $(\mathrm{u})$ soft gluons from collinear fields. At leading order we show explicitly that (u)soft gluon interactions cancel in the sum of all Feynman diagrams involving color singlet four quark operators. For the color octet operators we show that (u)soft gluons leave a color structure which vanishes between the physical singlet states.

In section $\mathrm{VB}$ we discuss $B \rightarrow X_{s} \gamma$ as an example of factorization in an inclusive decay. In this case the interesting region of phase space is $E_{\gamma} \gtrsim m_{B} / 2-\Lambda_{\mathrm{QCD}} \simeq 2.2 \mathrm{GeV}$, giving $\lambda=\sqrt{\Lambda_{\mathrm{QCD}} / Q}$. At leading order the rate near the photon endpoint can be factored into a hard coefficient multiplying a nontrivial convolution of a purely collinear function with a purely soft function. This was first shown in Ref. [7]. In this section we reproduce the

\footnotetext{
${ }^{2}$ This is similar to the multipole expansion for ultrasoft gluons in non-relativistic QCD [20], which is also necessary for a consistent power counting.
} 
proof of this result to all orders in perturbation theory using the effective theory. The nonperturbative soft function is the light cone structure function of the $B$ meson [6] $S\left(k^{+}\right)$. Since the collinear particles have offshellness $p^{2} \simeq Q \Lambda_{\mathrm{QCD}}$, the collinear function can be calculated perturbatively with a light-cone operator product expansion. At leading order the decay rate is then given by a calculable function convoluted with $S\left(k^{+}\right)$.

\section{WILSON LINES IN HQET}

Constructing and understanding the SCET requires the introduction of Wilson lines along various light-like paths. In this section we introduce some of the concepts using the well known example of heavy quark effective theory. The physics for heavy quarks such as bottom and charm can be described in a systematic way by expanding about the infinite mass limit, $m_{b}, m_{c} \rightarrow \infty$. The standard leading HQET Lagrangian and heavy-to-heavy current are [16]

$$
\mathcal{L}_{\mathrm{HQET}}=\sum_{v} \bar{b}_{v} i v \cdot D b_{v}+\sum_{v^{\prime}} \bar{c}_{v^{\prime}} i v^{\prime} \cdot D c_{v^{\prime}}, \quad J_{v, v^{\prime}}=\bar{c}_{v^{\prime}} \Gamma b_{v},
$$

where $\Gamma$ is the spin structure. Here $b_{v}$ and $c_{v^{\prime}}$ are effective theory fields labelled by their velocity, and the covariant derivative $i D^{\mu}=i \partial^{\mu}+g A^{\mu}$ involves only soft gluons. This theory of static quarks is related to a theory of Wilson lines along directions specified by $v$ [23],

$$
S_{v}(x)=\mathrm{P} \exp \left(i g \int_{-\infty}^{x} \mathrm{~d} s v \cdot A(v s)\right) .
$$

The covariant derivative along the path of a Wilson line is zero, $v \cdot D S_{v}=0$, which is why Eq. (4) is referred to as a static Wilson line. Now consider defining new heavy quark fields $b_{v}^{(0)}$ and $c_{v^{\prime}}^{(0)}$ by

$$
b_{v}(x)=S_{v}(x) b_{v}^{(0)}(x), \quad c_{v^{\prime}}(x)=S_{v^{\prime}}(x) c_{v^{\prime}}^{(0)}(x) .
$$

Using $v \cdot D S_{v} b_{v}^{(0)}=S_{v} v \cdot \partial b_{v}^{(0)}$ and the unitarity condition $S_{v}^{\dagger} S_{v}=1$ the Lagrangian and current are then

$$
\mathcal{L}_{\mathrm{HQET}}=\sum_{v} \bar{b}_{v}^{(0)} i v \cdot \partial b_{v}^{(0)}+\sum_{v^{\prime}} \bar{c}_{v^{\prime}}^{(0)} i v^{\prime} \cdot \partial c_{v^{\prime}}^{(0)}, \quad J_{v, v^{\prime}}=\bar{c}_{v^{\prime}}^{(0)} S_{v^{\prime}}^{\dagger} \Gamma S_{v} b_{v}^{(0)} .
$$

The new fields $b_{v}^{(0)}$ and $c_{v^{\prime}}^{(0)}$ still annihilate heavy quarks, however they no longer interact with soft gluons. f All soft gluon interactions are explicit in the Wilson lines which appear in the heavy-to-heavy current.

\footnotetext{
${ }^{3}$ Note that $\mathcal{L}$ in Eq. (6) is still gauge invariant since under a soft QCD gauge transformation, $V(x)=\exp \left[i \alpha^{B}(x) T^{B}\right]$, the fields $b_{v}^{(0)}$ and $c_{v^{\prime}}^{(0)}$ do not transform. The current is gauge invariant since $S_{v} \rightarrow V S_{v}$ (with $\alpha^{B}(\infty)=0$ ). Using $V^{\dagger} V=1$ then gives $J_{v, v^{\prime}} \rightarrow J_{v, v^{\prime}}$.
} 
Since Eq. (3) and Eq. (6) are simply related by a field redefinition, the new Lagrangian and current describe the same physics as the original ones. For example, it is well known that the matrix element of $J_{v, v^{\prime}}$ between $B$ and $D$ states is the universal Isgur-Wise function 24]

$$
\left\langle D_{v^{\prime}}\left|J_{v, v^{\prime}}\right| \bar{B}_{v}\right\rangle=\operatorname{Tr}\left[\frac{\left(1+\psi^{\prime}\right)}{2} \Gamma \frac{(1+\psi)}{2}\right] \xi\left(v \cdot v^{\prime}\right)
$$

which is normalized at zero recoil, $\xi(1)=1$. For $v^{\prime}=v$, we see that all soft gluon interactions in $J_{v, v}$ in Eq. (6) cancel since $S_{v}^{\dagger} S_{v}=1$. This shows explicitly that with $v^{\prime} \cdot v=1$ and $m_{b, c} \rightarrow \infty$ the "brown muck" in the $B$ and $D$ does not observe the $b \rightarrow c$ transition.

In the remainder of the paper we will use the standard Lagrangian in Eq. (3) to describe heavy quark fields. However, the manipulations in this section allow us to draw simple parallels with our discussion of the SCET. In section III we will introduce the analog of the effective Lagrangian in Eq. (3) for interactions between usoft and collinear fields. Then in section $I \nabla$ we will show that collinear fields analogous to $b_{v}^{(0)}$ can be defined which do not couple to usoft gluons. Many statements about soft-collinear factorization in section $\nabla$ then simply follow from the unitarity condition which is analogous to $S_{v}^{\dagger} S_{v}=1$.

\section{SOFT-COLLINEAR EFFECTIVE THEORY}

We begin in section IIIA by recalling some basic ideas that go into the construction of the SCET [1] 3]. After describing the degrees of freedom and power counting, we summarize the general result for the structure of Wilson coefficients that can arise from integrating out hard fluctuations. Finally, we review why a collinear Wilson line appears in the effective theory. In section $\mathbb{1 1} \mathrm{B}$ the collinear, soft, and usoft gauge symmetries of the effective theory are discussed. In section $\mathbb{\amalg I} \mathrm{C}$ the leading order gauge invariant actions for collinear and (u)soft quarks and gluons are given.

\section{A. Basics}

The goal of the SCET is to describe interactions between energetic and non-energetic particles in a common frame of reference. The relevant momentum scales are $Q, Q \lambda$, and $Q \lambda^{2}$. Collinear modes are needed to describe fluctuations about the collinear momenta $Q\left(\lambda^{2}, 1, \lambda\right)$, while soft and usoft modes are needed to describe fluctuations about the $Q \lambda$ and $Q \lambda^{2}$ scales respectively. Other possible momenta such as $p^{\mu} \sim Q(1,1,1)$ and $p^{\mu} \sim Q(\lambda, 1, \lambda)$ are integrated out since they describe offshell fluctuations. In Table — the effective theory quark and gluon fields are given along with their power counting in $\lambda$. The power counting is assigned such that in the action the kinetic terms for these fields are order $\lambda^{0}$. For instance, for an usoft gluon setting $\int d^{4} x_{u s} A_{u s} \partial^{2} A_{u s} \sim \lambda^{0}$ and using $d^{4} x_{u s} \sim \lambda^{-8}$ and $\partial^{2} \sim \lambda^{4}$ gives $A_{u s}^{\mu} \sim \lambda^{2}$. 


\begin{tabular}{|c|c|c|c|}
\hline Type & Momenta $p^{\mu}=\left(p^{+}, p^{-}, p^{\perp}\right)$ & Fields & Field Scaling \\
\hline \multirow[t]{3}{*}{ collinear } & $p^{\mu} \sim\left(\lambda^{2}, 1, \lambda\right)$ & $\xi_{n, p}$ & $\lambda$ \\
\hline & & $\left(A_{n, p}^{+}, A_{n, p}^{-}, A_{n, p}^{\perp}\right)$ & $\left(\lambda^{2}, 1, \lambda\right)$ \\
\hline & & $W\left[\bar{n} \cdot A_{n, p}\right]$ & 1 \\
\hline \multirow[t]{3}{*}{ soft } & $p^{\mu} \sim(\lambda, \lambda, \lambda)$ & $q_{s, p}$ & $\lambda^{3 / 2}$ \\
\hline & & $A_{s, p}^{\mu}$ & $\lambda$ \\
\hline & & $S\left[n \cdot A_{s, p}\right]$ & 1 \\
\hline \multirow[t]{3}{*}{ usoft } & $k^{\mu} \sim\left(\lambda^{2}, \lambda^{2}, \lambda^{2}\right)$ & $q_{u s}$ & $\lambda^{3}$ \\
\hline & & $A_{u s}^{\mu}$ & $\lambda^{2}$ \\
\hline & & $Y\left[n \cdot A_{u s}\right]$ & 1 \\
\hline
\end{tabular}

TABLE I. Power counting for the effective theory quarks and gluons. The Wilson lines $W, S$, and $Y$ are defined in Eqs. (13-15).

In constructing the effective theory a separation of momentum scales is achieved by decomposing the full momentum as $P^{\mu}=p^{\mu}+k^{\mu}$, where $p^{\mu}$ is a label containing momenta of order $Q$ and $Q \lambda$, while the residual momentum $k^{\mu}$ scales as $Q \lambda^{2}$. In the fields the large phases depending on $p$ are removed, and this momentum becomes a label on the effective theory field [2,3]. For instance, for the collinear gluon field $A_{n, p}^{\mu}$ one takes

$$
A^{\mu}(x) \rightarrow \sum_{p} e^{-i p \cdot x} A_{n, p}^{\mu}(x) .
$$

Similarly the collinear quark fields $\xi_{n, p}(x)$ have a momentum label $p$, and furthermore satisfy $\not h \xi_{n, p}=0$. It is convenient to define an operator $\mathcal{P}^{\mu}$ which acts on fields with labels,

$$
\mathcal{P}^{\mu}\left(\phi_{q_{1}}^{\dagger} \cdots \phi_{q_{m}}^{\dagger} \phi_{p_{1}} \cdots \phi_{p_{n}}\right)=\left(p_{1}^{\mu}+\ldots+p_{n}^{\mu}-q_{1}^{\mu}-\ldots-q_{m}^{\mu}\right)\left(\phi_{q_{1}}^{\dagger} \cdots \phi_{q_{m}}^{\dagger} \phi_{p_{1}} \cdots \phi_{p_{n}}\right) .
$$

This operator enables us to write $i \partial^{\mu} e^{-i p \cdot x} \phi_{p}(x)=e^{-i p \cdot x}\left(\mathcal{P}^{\mu}+i \partial^{\mu}\right) \phi_{p}(x)$. Thus, all large phases can be pulled to the front of any operator, and the remaining derivatives give only the residual $\sim Q \lambda^{2}$ momentum. Label sums and phases can be suppressed if we simply remember to conserve label momenta in interactions. For convenience we define the operator $\overline{\mathcal{P}}$ to pick out only the order $\lambda^{0}$ labels $\bar{n} \cdot p$ on collinear effective theory fields, and the operator $\mathcal{P}^{\mu}$ to pick out only the order $\lambda$ labels. Thus, for soft fields $\mathcal{P}^{\mu}$ gives the full momentum of the field, $\mathcal{P}^{\mu} q_{s, p}=p^{\mu} q_{s, p}$.

In the effective theory hard fluctuations are integrated out and appear in Wilson coefficients. Beyond tree level, these Wilson coefficients are nontrivial functions $C\left(\bar{n} \cdot p_{i}\right)$ of the 


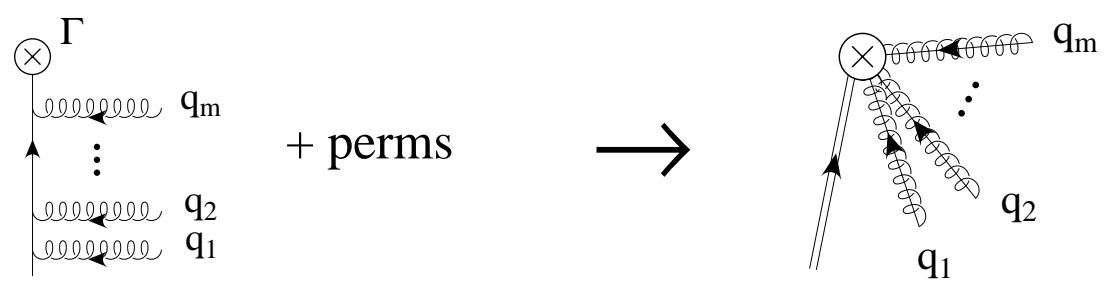

FIG. 1. A matching calculation which shows how $W$ appears. On the left collinear $\bar{n} \cdot A_{n, q}$ gluons hit an incoming soft quark. Integrating out the offshell quark propagators gives the effective theory operator on the right which contains a factor of $W$.

large collinear momenta. However, collinear gauge invariance restricts these coefficients to only depend on the linear combination picked out by the operator $\overline{\mathcal{P}}$ [3]. Thus, in general Wilson coefficients are functions $C\left(\overline{\mathcal{P}}, \overline{\mathcal{P}}^{\dagger}\right)$ which must be inserted between gauge invariant products of collinear fields.

From Table [1 we notice that the $\bar{n} \cdot A_{n, p}$ collinear gluon field is order $\lambda^{0}$ in the power counting. These gluons play a special role in the effective theory, since at a given order in $\lambda$ any number of them can appear. However, as described in Ref. [3] collinear gauge symmetry restricts them to only appear in the Wilson line functional

$$
W=\left[\sum_{\text {perms }} \exp \left(-g \frac{1}{\overline{\mathcal{P}}} \bar{n} \cdot A_{n, q}\right)\right] .
$$

Thus, in the effective theory $W$ should simply be treated as a basic building block for constructing operators. Gauge invariant combinations of $\bar{n} \cdot A_{n, q}$ can be written entirely in terms of $W$ since

$$
f\left(\overline{\mathcal{P}}+g \bar{n} \cdot A_{n, q}\right)=W f(\overline{\mathcal{P}}) W^{\dagger}
$$

Alternatively, we can view factors of $W$ as arising from having integrated out offshell propagators [2], as in Fig. 1. The figure illustrates that the inability of collinear gluons to interact with softer particles in a local manner is what leads to the appearance of $W$. Written out explicitly the Wilson line in Eq. (10) is

$$
W=\sum_{m=0}^{\infty} \sum_{\text {perms }} \frac{(-g)^{m}}{m !} \frac{\bar{n} \cdot A_{n, q_{1}}^{a_{1}} \cdots \bar{n} \cdot A_{n, q_{m}}^{a_{m}}}{\bar{n} \cdot q_{1} \bar{n} \cdot\left(q_{1}+q_{2}\right) \cdots \bar{n} \cdot\left(\sum_{i=1}^{m} q_{i}\right)} T^{a_{m}} \cdots T^{a_{1}}
$$

In the power counting $W \sim \lambda^{0}$. If we drop the dependence on $x$ (i.e. the residual momenta) in Eq. (12) and take the Fourier transform of the $\bar{n} \cdot q_{i}$ labels we obtain a Wilson line in position space

$$
W_{n, y}=\mathrm{P} \exp \left(i g \int_{-\infty}^{y} \mathrm{~d} s \bar{n} \cdot A_{n}(s \bar{n})\right)
$$

Here the position space field $A_{n}^{\mu}(z)$ is the Fourier transform of $A_{n, p}^{\mu}(0)$ with respect to $\bar{n} \cdot p$.

In Table [ two more eikonal lines appear which will be used later in the paper. The functional $S$ is built out of soft fields 


$$
S(x)=\mathrm{P} \exp \left(i g \int_{-\infty}^{x} \mathrm{~d} s n \cdot A_{s}(s n)\right)
$$

which vary over scales of order $Q \lambda$, and $Y$ is the analogous functional built out of usoft fields

$$
Y(x)=\mathrm{P} \exp \left(i g \int_{-\infty}^{x} \mathrm{~d} s n \cdot A_{u s}(s n)\right)
$$

which vary over scales of order $Q \lambda^{2}$. Unlike $W$, neither $S$ nor $Y$ are necessary to construct the Lagrangian for $(\mathrm{u})$ soft or collinear fields. However, both turn out to be useful in understanding how $(\mathrm{u})$ soft-collinear factorization arises as a property of the effective theory at lowest order.

\section{B. Gauge Symmetries in the SCET}

The presence of several gluon modes raises the question of how each is related to local transformations from the gauge group SU(3). Of the possible QCD gauge transformations the ones that are relevant to constructing the effective theory have support over collinear, soft, or usoft momenta. An usoft gauge transformation $V_{u s}(x)=\exp \left[i \beta_{u s}^{A}(x) T^{A}\right]$ is defined as the subset of gauge transformations where $\partial^{\mu} V_{u s}(x) \sim Q \lambda^{2}$. A soft gauge transformation $V_{s}(x)=\exp \left[i \beta_{s}^{A}(x) T^{A}\right]$ satisfies $\partial^{\mu} V_{s}(x) \sim Q \lambda$. Finally, collinear gauge transformations $U(x)=\exp \left[i \alpha^{A}(x) T^{A}\right]$ are the subset where $\partial^{\mu} U(x) \sim Q\left(\lambda^{2}, 1, \lambda\right)$. The usoft, soft, and collinear gluon fields are then the gauge fields associated with these transformations. The gauge transformations for the effective theory fields are shown in Table $\llbracket$. The physics that restricts the transformations of fields from one momentum region with respect to the gauge symmetry of another region are discussed below. We discuss the usoft, soft, and collinear transformations in turn.

Invariance under usoft gauge transformations constrain the self interactions of usoft gluons as well as their coupling to collinear fields. The slowly varying usoft gluon field acts like a classical background field in which the collinear and soft particles propagate. Under a usoft gauge transformation, $V_{u s}(x)$, the usoft quarks and gluons transform the same as in QCD. The collinear and soft particles have larger momenta, so they see the usoft gauge transformation as a smooth change in the background, and transform similar to a global color rotation. For soft fields all momentum components are larger than the usoft momenta so the transformations are effectively global. For collinear quarks and gluons the usoft transformations are local at each point of the residual $x$ dependence.

Soft gauge transformations have support over a region of momenta which leave neither usoft or collinear particles near their mass shell. The usoft and collinear fields do not transform since they cannot resolve the local change induced by $V_{s}(x)$. Therefore, soft gluons do not appear in the Lagrangians for collinear or usoft particles. On the other hand soft fields transform with $V_{s}$ like fields in QCD. We will see in section IVB that the gauge invariance of operators with soft and collinear fields requires factors of $S$ to appear. 


\begin{tabular}{|c|c|c|c|}
\hline & \multicolumn{3}{|c|}{ Gauge Transformations } \\
\hline Fields & Collinear $\mathcal{U}_{R}$ & Soft $V_{s}$ & Usoft $V_{u s}$ \\
\hline$\xi_{n, p}$ & $\mathcal{U}_{p-Q} \xi_{n, Q}$ & $\xi_{n, p}$ & $V_{u s} \xi_{n, p}$ \\
\hline$A_{n, p}^{\mu}$ & $\mathcal{U}_{Q} A_{n, R}^{\mu} \mathcal{U}_{Q+R-p}^{\dagger}+\frac{1}{g} \mathcal{U}_{Q}\left[i \mathcal{D}^{\mu} \mathcal{U}_{Q-p}^{\dagger}\right]$ & $A_{n, p}^{\mu}$ & $V_{u s} A_{n, p}^{\mu} V_{u s}^{\dagger}$ \\
\hline$q_{s}$ & $q_{s}$ & $V_{s} q_{s}$ & $V_{u s} q_{s}$ \\
\hline$A_{s}^{\mu}$ & $A_{s}^{\mu}$ & $V_{s}\left(A_{s}^{\mu}+\frac{1}{g} \mathcal{P}^{\mu}\right) V_{s}^{\dagger}$ & $V_{u s} A_{s}^{\mu} V_{u s}^{\dagger}$ \\
\hline$q_{u s}$ & $q_{u s}$ & $q_{u s}$ & $V_{u s} q_{u s}$ \\
\hline$A_{u s}^{\mu}$ & $A_{u s}^{\mu}$ & $A_{u s}^{\mu}$ & $V_{u s}\left(A_{u s}^{\mu}+\frac{i}{g} \partial^{\mu}\right) V_{u s}^{\dagger}$ \\
\hline \multicolumn{4}{|l|}{ Wilson Lines } \\
\hline$W$ & $\mathcal{U}_{Q} W$ & $W$ & $V_{u s} W V_{u s}^{\dagger}$ \\
\hline$S$ & $S$ & $V_{s} S$ & $V_{u s} S V_{u s}^{\dagger}$ \\
\hline$Y$ & $Y$ & $Y$ & $V_{u s} Y$ \\
\hline
\end{tabular}

TABLE II. Gauge transformations for the collinear, soft, and usoft fields and the Wilson lines $W, S$, and $Y$. The $p$ labels on collinear fields are fixed, while $Q$ and $R$ are summed over. For simplicity labels on the soft fields are suppressed here.

For a collinear gauge transformation $U(x)$ we factor out the large momenta just as was done for collinear fields,

$$
U(x)=\sum_{R} e^{-i R \cdot x} \mathcal{U}_{R}(x)
$$

where $\partial^{\mu} \mathcal{U}_{R} \sim \lambda^{2}$. As explained above, the usoft gluons act like a background field for the collinear particles. Thus, in the presence of usoft gluons the collinear gluon field transforms like a quantum field in a background color field. The covariant derivative appearing in the transformation of the collinear gluon in Table [1 is therefore

$$
i \mathcal{D}^{\mu} \equiv \frac{n^{\mu}}{2} \overline{\mathcal{P}}+\mathcal{P}_{\perp}^{\mu}+\frac{\bar{n}^{\mu}}{2} i n \cdot D
$$

\footnotetext{
${ }^{4}$ If $\mathcal{U}_{R}$ is independent of $x$, the collinear transformations are given in Ref. [3] and with

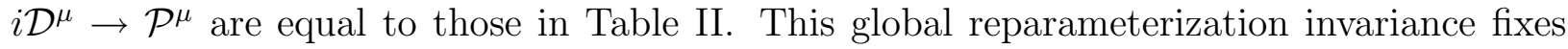
the collinear action, except for the way in which $i n \cdot D$ appears.
} 
Here $D^{\mu}$ contains only the usoft field

$$
i D^{\mu}=i \partial^{\mu}+g A_{u s}^{\mu}
$$

and by power counting only the $n \cdot A_{\text {us }}$ gluons can appear in Eq. (17) at leading order in $\lambda$ (since they are the same order as $n \cdot A_{n, p}$ ). Under a collinear gauge transformation the (u)soft quarks and gluons do not transform since they fluctuate over wavelengths which can not resolve the fast local change induced by $U(x)$.

\section{The Effective Lagrangian}

In this section the gauge properties discussed in section [IIB are used to construct the SCET Lagrangian. The full Lagrangian can be broken up into terms involving soft fields $\mathcal{L}_{s}$, terms involving collinear fields $\mathcal{L}_{c}$, and terms with neither soft nor collinear fields $\mathcal{L}_{u s}$,

$$
\mathcal{L}=\mathcal{L}_{s}+\mathcal{L}_{c}+\mathcal{L}_{u s}
$$

Invariance under usoft gauge transformations forces all $A_{u s}^{\mu}$ fields to appear through the covariant derivative $i D^{\mu}$ defined in Eq. (18). Invariance under soft gauge transformations constrain the appearance of the soft gauge field. Finally, from invariance under collinear gauge transformations the collinear gluon fields can only appear in factors of $W$ and

$$
i \mathcal{D}^{\mu}+g A_{n, q}^{\mu}
$$

where $\mathcal{D}^{\mu}$ is defined in Eq. (17). Under a collinear gauge transformation this operator transforms as

$$
i \mathcal{D}^{\mu}+g A_{n, q}^{\mu} \rightarrow \mathcal{U}_{Q} g A_{n, R}^{\mu} \mathcal{U}_{Q+R-q}^{\dagger}+\mathcal{U}_{Q} i \mathcal{D}^{\mu} \mathcal{U}_{Q-q}^{\dagger}
$$

The purely usoft and soft Lagrangians for gluons and massless quarks are the same as those in QCD and are determined uniquely by power counting and invariance under (u)soft gauge transformations.

$$
\begin{aligned}
\mathcal{L}_{u s} & =\bar{q}_{u s} i \not D q_{u s}-\frac{1}{2} \operatorname{tr}\left\{G^{\mu \nu} G_{\mu \nu}\right\}, \\
\mathcal{L}_{s} & =\bar{q}_{s, p^{\prime}}\left(\not \mathcal{P}+g A_{s, q}\right) q_{s, p}-\frac{1}{2} \operatorname{tr}\left\{G_{s}^{\mu \nu} G_{\mu \nu}^{s}\right\}
\end{aligned}
$$

where $G^{\mu \nu}=i\left[D^{\mu}, D^{\nu}\right] / g$ and $i G_{s}^{\mu \nu}=\left[\mathcal{P}^{\mu}+g A_{s, q}^{\mu}, \mathcal{P}^{\nu}+g A_{s, q^{\prime}}^{\nu}\right] / g$. The traces are normalized such that $\operatorname{tr}\left[T^{A} T^{B}\right]=\delta^{A B} / 2$. Gauge fixing terms for the usoft and soft gluons are not specified, and can be freely chosen without affecting the couplings of other modes. For heavy quarks we have the leading order HQET Lagrangian

$$
\mathcal{L}_{\mathrm{HQET}}=\sum_{v} \bar{h}_{v} i v \cdot D h_{v}
$$




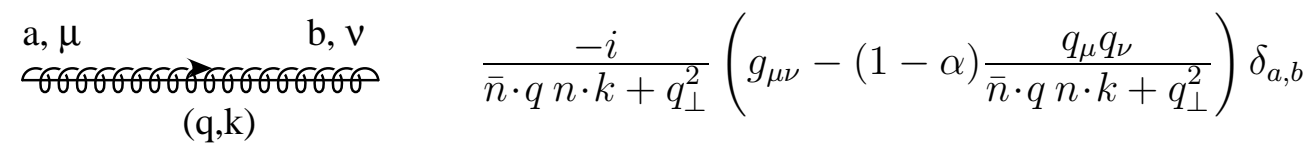
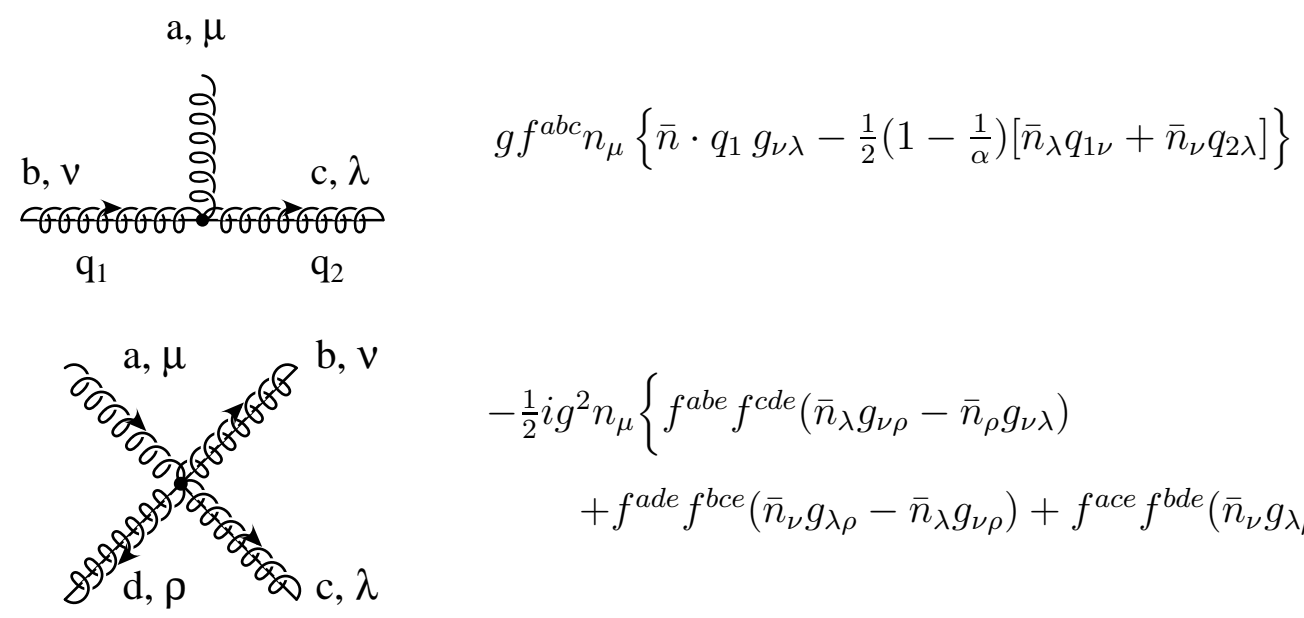

$$
\begin{aligned}
& -\frac{1}{2} i g^{2} n_{\mu}\left\{f^{a b e} f^{c d e}\left(\bar{n}_{\lambda} g_{\nu \rho}-\bar{n}_{\rho} g_{\nu \lambda}\right)\right. \\
& \left.\quad+f^{a d e} f^{b c e}\left(\bar{n}_{\nu} g_{\lambda \rho}-\bar{n}_{\lambda} g_{\nu \rho}\right)+f^{a c e} f^{b d e}\left(\bar{n}_{\nu} g_{\lambda \rho}-\bar{n}_{\rho} g_{\nu \lambda}\right)\right\}
\end{aligned}
$$

$\mathrm{a}, \mu \quad \mathrm{b}, \mathrm{v}$

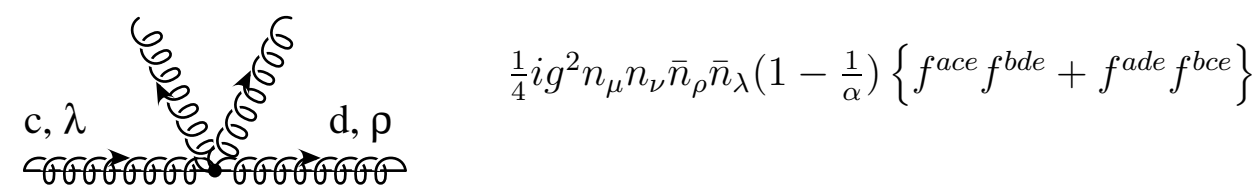

FIG. 2. Collinear gluon propagator with label momentum $q$ and residual momentum $k$, and the order $\lambda^{0}$ interactions of collinear gluons with the usoft gluon field. Here usoft gluons are springs, collinear gluons are springs with a line, and $\alpha$ is the covariant gauge fixing parameter in Eq. (24).

Here $h_{v}$ is defined as the flavor doublet of the fields $\left(b_{v}, c_{v}\right)$ used in section प1. For the Lagrangians in Eqs. (22) and (23), no additional information is gained from collinear gauge invariance since the $(\mathrm{u})$ soft fields do not transform.

For collinear gluons the gauge fixing terms are nontrivial since they affect how collinear gluons interact with the background usoft gluons. For simplicity we will use a general covariant gauge, which causes collinear ghosts fields $c_{n, q}$ to appear. At order $\lambda^{0}$ the Lagrangian for collinear gluons and ghosts is then

$$
\begin{aligned}
\mathcal{L}_{c}^{(g)}= & \frac{1}{2 g^{2}} \operatorname{tr}\left\{\left[i \mathcal{D}^{\mu}+g A_{n, q}^{\mu}, i \mathcal{D}^{\nu}+g A_{n, q^{\prime}}^{\nu}\right]\right\}^{2}+2 \operatorname{tr}\left\{\bar{c}_{n, p^{\prime}}\left[i \mathcal{D}_{\mu},\left[i \mathcal{D}^{\mu}+g A_{n, q}^{\mu}, c_{n, p}\right]\right]\right\} \\
& +\frac{1}{\alpha} \operatorname{tr}\left\{\left[i \mathcal{D}_{\mu}, A_{n, q}^{\mu}\right]\right\}^{2},
\end{aligned}
$$

where $\mathcal{D}^{\mu}$ is defined in Eq. (17). Note that $A_{u s}^{\perp}$ and $\bar{n} \cdot A_{u s}$ do not appear until higher order since they are suppressed compared to the collinear gluon fields. The $n \cdot A_{u s}$ component can appear since it is the same order as $n \cdot A_{n, q}$. The first two terms in Eq. (24) are invariant under collinear gauge transformations, while the last is the gauge fixing term with parameter $\alpha$. Furthermore, the complete collinear Lagrangian is invariant under usoft gauge 
transformations, including the $1 / \alpha$ gauge fixing term. This complies with the notion of the usoft gluon as a background field. In fact, Eq. (24) is identical to the action obtained by expanding the covariant background field action in QCD with quantum field $A_{n, q}^{\mu}$ and background field $A_{u s}^{\mu}$ to leading order in $\lambda$. The Feynman rules for collinear gluon self interactions are the same as those in QCD except that the residual $k^{\perp}$ and $\bar{n} \cdot k$ momenta do not appear. The mixed usoft-collinear Feynman rules that follow from Eq. (24) are more interesting and are shown in Fig. 2.

For completeness, we also give the collinear quark Lagrangian [2,3] which is derived in a similar mannerf

$$
\mathcal{L}_{c}^{(q)}=\bar{\xi}_{n, p^{\prime}}\left\{i n \cdot D+g n \cdot A_{n, q}+\left(\not{P}_{\perp}+g A_{n, q}^{\perp}\right) W \frac{1}{\overline{\mathcal{P}}} W^{\dagger}\left(\not{P}_{\perp}+g \mathcal{A}_{n, q^{\prime}}^{\perp}\right)\right\} \frac{\not h}{2} \xi_{n, p} .
$$

The terms involving gluons in Eq. (25) are simply components of $i \mathcal{D}^{\mu}+g A_{n, q}^{\mu}$, as required by gauge invariance. $\mathcal{L}_{c}^{(q)}$ is the unique order $\lambda^{0}$ collinear quark Lagrangian that is invariant under both collinear and usoft gauge transformations.

\section{COLLINEAR FIELDS INTERACTING WITH (U)SOFT GLUONS}

In this section we present a way of organizing the couplings of $(\mathrm{u})$ soft gluons to collinear particles which makes factorization properties more transparent by moving interaction vertices into operators. The traditional method of proving the factorization of (u)soft gluons uses reduced graphs and eikonal Ward identities [5]. While our approach makes use of similar physical observations, we believe that the SCET organizes these properties in a simpler way. For example, in the effective theory the multipole expansion for usoft couplings and appearance of only $n \cdot A_{u s}$ gluons is explicit in the collinear Lagrangian. We start by discussing usoft gluon couplings to collinear fields in section IVA. At lowest order in $\lambda$, the couplings of usoft gluons to collinear quarks and gluons can be summed into Wilson lines which act like field valued gauge rotations. Similar to the $b_{v}^{(0)}$ heavy quarks in section [1, the new collinear fields $\xi_{n, p}^{(0)}$ and $A_{n, p}^{(0) \mu}$ no longer couple to usoft gluons. In section IVB and Appendix $\mathrm{A}$ we discuss the factorization for soft modes.

\section{A. Ultrasoft couplings to collinear quarks and gluons}

Consider the interaction of the collinear fields with an usoft background gluon field. The relevant diagrams are shown in Figs. 3 and 4 . Matching on-shell, the sum of the diagrams

\footnotetext{
${ }^{5}$ Apart from the coupling to usoft gluons and the treatment of residual momenta the collinear quark action is similar to the action for a quark in light-cone quantization [25]. Given the equivalence [26] of QCD quantized on the light-cone and QCD in the infinite momentum frame this is not too surprising.
} 


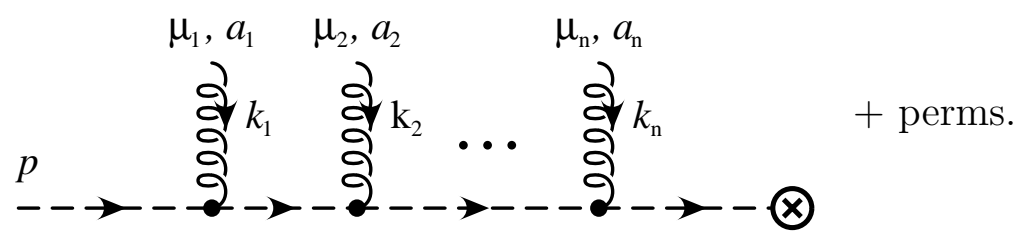

FIG. 3. The attachments of usoft gluons to a collinear quark line which are summed up into a path-ordered exponential.

which couple usoft gluons to the collinear quark give

$$
\xi_{n, p}=Y \xi_{n, p}^{(0)}
$$

where

$$
Y=1+\sum_{m=1}^{\infty} \sum_{\text {perms }} \frac{(-g)^{m}}{m !} \frac{n \cdot A_{u s}^{a_{1}} \cdots n \cdot A_{u s}^{a_{m}}}{n \cdot k_{1} n \cdot\left(k_{1}+k_{2}\right) \cdots n \cdot\left(\sum_{i=1}^{m} k_{i}\right)} T^{a_{m}} \cdots T^{a_{1}}
$$

$Y$ is related to the Fourier transform of the path-ordered exponential given in Eq. (15),

$$
Y(x)=\mathrm{P} \exp \left(i g \int_{-\infty}^{x} \mathrm{~d} s n \cdot A_{u s}^{a}(n s) T^{a}\right) .
$$

In Eq. (26) the new collinear quark field $\xi_{n, p}^{(0)}$ does not interact with usoft gluons. Thus, all interactions with usoft gluons have been summed into the Wilson line. Although Eq. (26) was derived at tree level, the presence of soft or collinear loops does not change this result. We will prove this at the level of the action near the end of this section.

In a similar manner, we can compute the sum of the diagrams which couple usoft gluons to a collinear gluon shown in Fig. 6 . Using the Feynman rules in Fig. 2 in Feynman gauge $(\alpha=1)$ gives

$$
A_{n, p}^{a, \mu}=\mathcal{Y}^{a b} A_{n, p}^{(0) b, \mu}
$$

where

$$
\mathcal{Y}^{a b}=\delta^{a b}+\sum_{m=1}^{\infty} \sum_{\text {perms }} \frac{(i g)^{m}}{m !} \frac{n \cdot A_{u s}^{a_{1}} \cdots n \cdot A_{u s}^{a_{m}}}{n \cdot k_{1} n \cdot\left(k_{1}+k_{2}\right) \cdots n \cdot\left(\sum_{i=1}^{m} k_{i}\right)} f^{a_{m} a x_{m-1}} \cdots f^{a_{2} x_{2} x_{1}} f^{a_{1} x_{1} b} .
$$

Similar to the quark field, $A_{n, p}^{(0)}$ denotes a collinear gluon which does not couple to usoft gluons. The result in Eq. (29) is gauge invariant with respect to the usoft gluons because of the usoft invariance of the collinear gluon Lagrangian. Thus, in deriving this result it is crucial that the derivative in the $1 / \alpha$ gauge fixing term in Eq. (24) is covariant with respect

${ }^{6}$ In other gauges the derivation is complicated by the presence of the four-gluon vertex, and the need to use $p \cdot A_{n, p}=0$ for the collinear gluon field. 


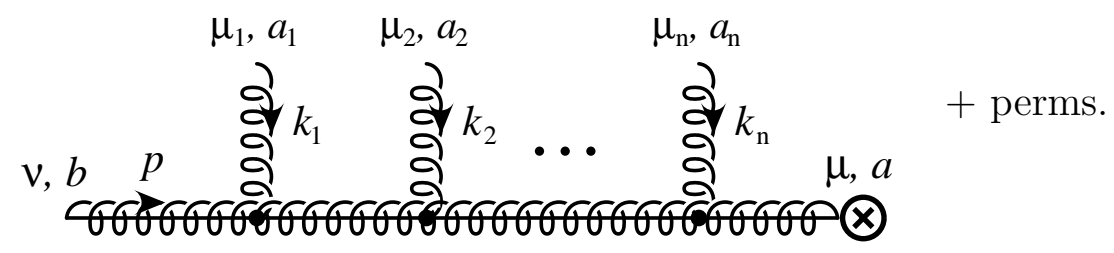

FIG. 4. The attachments of usoft gluons to a collinear gluon which are also summed up into a path-ordered exponential.

to the usoft $n \cdot A_{u s}$ background field. The $\mathcal{Y}^{a b}$ in Eq. (30) is related to the Fourier transform of the Wilson line $Y(x)$ in the adjoint representation

$$
\mathcal{Y}^{a b}(x)=\left[\mathrm{P} \exp \left(i g \int_{-\infty}^{x} \mathrm{~d} s n \cdot A_{u s}^{e}(n s) \mathcal{T}^{e}\right)\right]^{a b},
$$

where $\left(\mathcal{T}^{e}\right)^{a b}=-i f^{e a b}$. The adjoint representation can be defined in terms of the fundamental representation by

$$
Y T^{a} Y^{\dagger}=\mathcal{Y}^{b a} T^{b}
$$

From this result we immediately obtain that

$$
A_{n, p}^{\mu}=A_{n, p}^{b, \mu} T^{b}=A_{n, p}^{(0) a, \mu} \mathcal{Y}^{b a} T^{b}=A_{n, p}^{(0) a, \mu} Y T^{a} Y^{\dagger}=Y A_{n, p}^{(0) \mu} Y^{\dagger}
$$

Repeating the above calculation for the collinear ghost field we find

$$
c_{n, p}=c_{n, p}^{a} T^{a}=c_{n, p}^{(0) b} \mathcal{Y}^{a b} T^{a}=Y c_{n, p}^{(0)} Y^{\dagger}
$$

Recalling that $A_{n, p}$ and hence $W$ are local with respect to their residual momentum, they are therefore local with respect to the coordinate $x$ of $Y(x)$. Thus,

$$
W=\left[\sum_{\text {perms }} \exp \left(-g \frac{1}{\overline{\mathcal{P}}} Y \bar{n} \cdot A_{n, q}^{(0)} Y^{\dagger}\right)\right]=Y W^{(0)} Y^{\dagger}
$$

which shows how usoft gluons couple to the collinear Wilson line.

Finally, we return to the claim that the field redefinitions in Eqs. (27) and (29) which define $\xi_{n, p}^{(0)}$ and $A_{n, p}^{(0)}$, decouple usoft gluons at the level of the Lagrangian. Starting with the collinear quark Lagrangian in Eq. (25), we obtain

$$
\begin{aligned}
\mathcal{L}_{c}^{(q)}=\bar{\xi}_{n, p^{\prime}}^{(0)} Y^{\dagger}\left\{i n \cdot D+g Y n \cdot A_{n, q}^{(0)} Y^{\dagger}+\left(\mathcal{P}_{\perp}+Y g A_{n, q}^{(0) \perp} Y^{\dagger}\right) Y W^{(0)} Y^{\dagger} \frac{1}{\overline{\mathcal{P}}}\right. \\
\left.\left.\times Y W^{(0) \dagger} Y^{\dagger}\left(\mathcal{P}_{\perp}+Y g A_{n, q^{\prime}}^{(0) \perp} Y^{\dagger}\right)\right\} \frac{\not h}{2} Y \xi_{n, p}^{(0)}\right\} \\
=\bar{\xi}_{n, p^{\prime}}^{(0)}\left\{Y^{\dagger} i n \cdot D Y+g n \cdot A_{n, q}^{(0)}+\left(\not{P}_{\perp}+g A_{n, q}^{(0) \perp}\right) W^{(0)} \frac{1}{\overline{\mathcal{P}}} W^{(0) \dagger}\left(\mathcal{P}_{\perp}+g A_{n, q^{\prime}}^{(0) \perp}\right)\right\} \frac{\not \hbar}{2} \xi_{n, p}^{(0)},
\end{aligned}
$$

since $Y$ commutes with $\mathscr{P}_{\perp}$. Using $n \cdot D Y=0$ it then follows that 


$$
Y^{\dagger} n \cdot D Y=n \cdot \partial
$$

Therefore, the collinear quark Lagrangian becomes

$$
\mathcal{L}_{c}^{(q)}=\bar{\xi}_{n, p^{\prime}}^{(0)}\left\{i n \cdot \partial+g n \cdot A_{n, q}^{(0)}+\left(\not{P}_{\perp}+g A_{n, q}^{(0) \perp}\right) W^{(0)} \frac{1}{\overline{\mathcal{P}}} W^{(0) \dagger}\left(\not{P}_{\perp}+g A_{n, q^{\prime}}^{(0) \perp}\right)\right\} \frac{\not \hbar}{2} \xi_{n, p}^{(0)},
$$

which is completely independent of the usoft gluon field. In a similar fashion the collinear gluon Lagrangian becomes

$$
\begin{aligned}
\mathcal{L}_{c}^{(g)}= & \frac{1}{2 g^{2}} \operatorname{tr}\left\{\left[i \mathcal{D}_{(0)}^{\mu}+g A_{n, q}^{(0) \mu}, i \mathcal{D}_{(0)}^{\nu}+g A_{n, q^{\prime}}^{(0) \nu}\right]\right\}^{2}+\frac{1}{\alpha} \operatorname{tr}\left\{\left[i \mathcal{D}_{\mu}^{(0)}, A_{n, q}^{(0) \mu}\right]\right\}^{2} \\
& +2 \operatorname{tr}\left\{\bar{c}_{n, p^{\prime}}^{(0)}\left[i \mathcal{D}_{\mu}^{(0)},\left[i \mathcal{D}_{(0)}^{\mu}+g A_{n, q}^{(0) \mu}, c_{n, p}^{(0)}\right]\right]\right\}
\end{aligned}
$$

where

$$
i \mathcal{D}_{(0)}^{\mu}=\frac{n^{\mu}}{2} \overline{\mathcal{P}}+\mathcal{P}_{\perp}^{\mu}+\frac{\bar{n}^{\mu}}{2} i n \cdot \partial
$$

Eq. (39) is derived using $i \mathcal{D}^{\mu}+g A_{n, q}^{\mu}=Y\left(i \mathcal{D}_{(0)}^{\mu}+g A_{n, q}^{(0) \mu}\right) Y^{\dagger}$. The result in Eq. (39) shows that the new collinear gluon and ghost fields $A_{n, q}^{(0)}$ and $c_{n, p}^{(0)}$ also decouple from usoft gluons.

To summarize, we have shown that making the field redefinitions

$$
\xi_{n, p}=Y \xi_{n, p}^{(0)}, \quad A_{n, p}^{\mu}=Y A_{n, p}^{(0) \mu} Y^{\dagger}, \quad c_{n, p}=Y c_{n, p}^{(0)} Y^{\dagger}
$$

the new collinear fields no longer couple to usoft gluons through their kinetic term. This gives the important result that all couplings of usoft gluons to collinear particles can be absorbed into Wilson lines $Y$ along the direction of the collinear particles. With these field redefinitions factors of $Y$ only appear in external operators or currents which contain collinear fields. We hasten to add that this is a property of the SCET only at leading order in $\lambda$. Beyond leading order subleading couplings of usoft gluons appear in the collinear Lagrangian which can not be reproduced solely by factors of $Y$.

\section{B. Soft couplings to collinear quarks and gluons}

For processes with soft gluon degrees of freedom the situation is quite different from the usoft case. This is because soft gluons can not couple to collinear particles without taking them off their mass shell. Together with soft gauge invariance this ensures that soft gluons do not appear in the collinear Lagrangian, and must therefore be explicit in operators. When a soft particle interacts with a collinear particle, it produces an offshell particle with momentum $p \sim Q(\lambda, 1, \lambda)$. For example, a triple gluon vertex with a soft and collinear gluon has an offshell gluon with momentum $Q(\lambda, 1, \lambda)$ as shown in Fig. 5. These offshell modes have $p^{2} \sim Q^{2} \lambda \gg(Q \lambda)^{2}$ and can therefore be integrated out of the theory. 


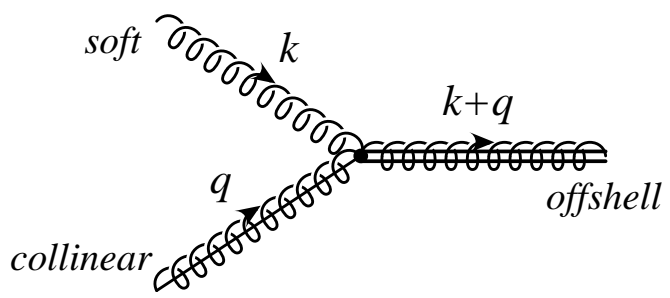

FIG. 5. The interaction of a soft and collinear gluon with momenta $k \sim Q(\lambda, \lambda, \lambda)$ and $q \sim Q\left(\lambda^{2}, 1, \lambda\right)$ respectively, to produce an offshell gluon with momentum $k+q \sim Q(\lambda, 1, \lambda)$.

There are several important properties that soft gluons obey. At lowest order in $\lambda$ soft interactions with collinear fields only involve the $n \cdot A_{s}$ component. Furthermore, integrating out all offshell fluctuations simply builds up factors of the Wilson line $S$,

$$
S=\left[\sum_{\text {perms }} \exp \left(-g \frac{1}{n \cdot \mathcal{P}} n \cdot A_{s, q}\right)\right] .
$$

where $\delta_{p, n \cdot \mathcal{P}} S$ is the Fourier transform of Eq. (14). Thus, much like $W, S$ turns out to be a fundamental object in the SCET. Finally, soft gauge invariance severely restricts the most general allowed operators involving $S$.

In this section we give an explicit example of the above properties and discuss how gauge invariance restricts the appearance of factors of $S$. In Appendix A we show in general that only $n \cdot A_{s}$ gluons appear at order $\lambda^{0}$. There we also show to all orders in perturbation theory how gauge invariant soft-collinear operators are obtained from integrating out offshell quarks and gluons. The proof is reduced to solving a classical two dimensional QCD action in the presence of adjoint and fundamental sources.

Consider the example of a soft-collinear heavy-to-light current. Under soft and collinear gauge transformations (suppressing the soft field labels) the fermions transform as

$$
\begin{array}{llll}
\text { soft: } & h_{v} \rightarrow V_{s} h_{v}, & \xi_{n, p} \rightarrow \xi_{n, p} \\
\text { collinear: } & h_{v} \rightarrow h_{v}, & \xi_{n, p} \rightarrow \mathcal{U}_{p-Q} \xi_{n, Q} .
\end{array}
$$

Thus, the simplest current $J=\bar{\xi}_{n, p} \Gamma h_{v}$ (where $\Gamma$ is the spin structure) is not invariant under the gauge symmetries. To construct a gauge invariant current requires the addition of soft and collinear Wilson lines. Using the transformation properties

$$
W \rightarrow \mathcal{U}_{Q} W, \quad S \rightarrow V_{s} S
$$

it is easy to see that the gauge invariant current is

$$
J=\bar{\xi}_{n, p} W \Gamma S^{\dagger} h_{v}
$$

Thus, we see that soft gauge invariance determines how $S$ appears.

It is also possible to obtain this current by matching, starting with the QCD current $\bar{q} \Gamma b$, and using background field gauge for the external gluons. There are three properties 
(a)

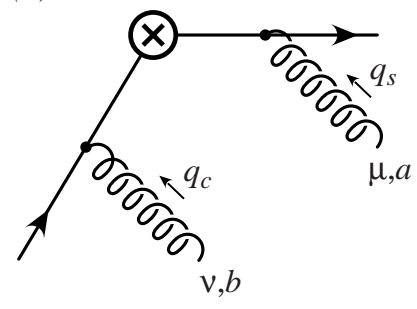

(b)

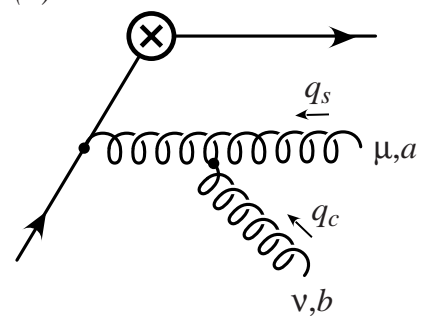

(c)

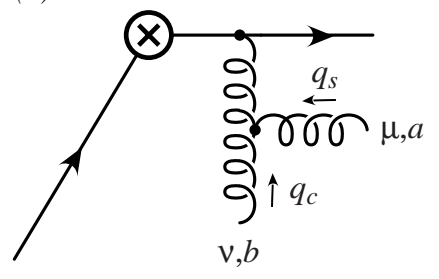

FIG. 6. QCD graphs for the current $\bar{q} \Gamma b$ with offshell propagators induced by a soft gluon with momentum $q_{s}$, and a collinear gluon with momentum $q_{c}$.

of Eq. (45) that need to be reproduced by this calculation, namely that only $\bar{n} \cdot A_{n, q}$ gluons appear to give $W$, that only the $n \cdot A_{s}$ component of the soft gluons appear to build up $S^{\dagger}$, and that $W$ and $S^{\dagger}$ appear in the gauge invariant combination shown. The calculation is similar to producing $W$ by attaching collinear gluons to heavy quarks and integrating out the resulting offshell fluctuations as discussed in section IIIA. Naively, attaching collinear gluons to the $b$ and soft gluons to the $q$, one might expect to build up the current $\bar{\xi}_{n, p} S^{\dagger} \Gamma W h_{v}$, and in the non-Abelian theory $S^{\dagger}$ and $W$ do not commute. However, adding diagrams involving non-Abelian gluon couplings reverses the order of the two Wilson lines.

For example, consider the order $g^{2}$ graphs which match onto Eq. (45) and which contain one soft and one collinear gluon. The necessary graphs are shown in Fig. 6.7 Expanding the diagram in Fig. 6a to leading order gives

$$
\text { Fig. } 6 \mathrm{a}=-g^{2} \frac{n^{\mu}}{n \cdot q_{s}} \frac{\bar{n}^{\nu}}{\bar{n} \cdot q_{c}} \bar{\xi}_{n, p} T^{a} \Gamma T^{b} h_{v} .
$$

We see that the leading contribution contains only the $\bar{n} \cdot A_{n, q} \sim \lambda^{0}$ collinear gluon by power counting, and only the $n \cdot A_{s}$ gluon because the $\gamma^{\nu}$ for this vertex is sandwiched between a $\bar{\xi}_{n, p}$ and an $\not h$ from the offshell light quark propagator. Finally, the color factors in Eq. (46) correspond to expanding $\bar{\xi}_{n, p} S^{\dagger} \Gamma W h_{v}$ or in other words are in exactly the opposite order to those in Eq. (45). At leading order the remaining two non-Abelian graphs are equal and give

$$
\text { Fig. } 6 \mathrm{~b}=\text { Fig. } 6 \mathrm{ac}=\frac{g^{2}}{2} i f^{a b c} T^{c} \frac{n^{\mu}}{n \cdot q_{s}} \frac{\bar{n}^{\nu}}{\bar{n} \cdot q_{c}} \bar{\xi}_{n, p} \Gamma h_{v} .
$$

Adding the three graphs together reverses the order of the color matrices in Eq. (46) to give

$$
\text { Figs. } 6 \mathrm{a}+\left[\mathrm{G} \mathrm{b}+6 \mathrm{G}=-g^{2} \frac{n^{\mu}}{n \cdot q_{s}} \frac{\bar{n}^{\nu}}{\bar{n} \cdot q_{c}} \bar{\xi}_{n, p} T^{b} \Gamma T^{a} h_{v} .\right.
$$

This is the desired result and is in agreement with Eq. (45). In Appendix $\mathrm{A}$ we extend this matching calculation to all orders in perturbation theory.

\footnotetext{
${ }^{7}$ Note that only graphs in which all propagators are offshell need to be considered for this matching.
} 


\section{APPLiCATIONS}

In this section we give two applications of our results. The factorization of soft and collinear modes has implications for the exclusive decays $B^{-} \rightarrow D^{0} \pi^{-}$and $B^{0} \rightarrow D^{+} \pi^{-}$, and is discussed in section $\nabla \mathrm{A}$. The SCET can be used to give a simple proof of the cancellation of nonfactorizable $(u)$ soft gluon effects at leading order in $\Lambda_{\mathrm{QCD}} / m_{b}$ [14], and this result is discussed using the notation introduced in Sec. III. The cancellation follows from gauge invariance and the unitarity of the Wilson line operators $S$ and $Y$.

In section $\mathrm{VB}$ we give a simple proof of the factorization formula for the photon spectrum in the end point region of the inclusive decay $B \rightarrow X_{s} \gamma$. This formula was first derived in Ref. [7]. In this region the photon spectrum can be written as a product of hard, usoft, and collinear factors, each of which have field-theoretical interpretations in the effective theory. In this case the nontrivial part is the factorization of usoft gluons. When the field redefinition for collinear fields is made in the time-ordered product, factors of the usoft Wilson line $Y$ appear at different space-time points and their cancellation is incomplete. They leave behind a finite Wilson line, which gives the light-cone structure function of the $B$ meson.

\section{A. $B \rightarrow D \pi$}

In this section we make use of the results in section $\mathbb{I V}$ to explain in detail why (u)soft gluons factor from the collinear particles in the pion for $B \rightarrow D \pi$. The proof is simplified by the fact that the cancellation of $(u)$ soft gluons appear at the level of the operators and the Lagrangian in the SCET.

At leading order the decay $B \rightarrow D \pi$ is mediated by the four-quark operators

$$
\mathcal{H}_{W}=\frac{4 G_{F}}{\sqrt{2}} V_{u d}^{*} V_{c b}\left[C_{0}^{F}\left(\bar{c} \gamma_{\mu} P_{L} b\right)\left(\bar{d} \gamma^{\mu} P_{L} u\right)+C_{8}^{F}\left(\bar{c} \gamma_{\mu} P_{L} T^{a} b\right)\left(\bar{d} \gamma^{\mu} P_{L} T^{a} u\right)\right]
$$

where $P_{L}=\frac{1}{2}\left(1-\gamma_{5}\right)$ and the coefficients $C_{0,8}^{F}$ are obtained by running down from the weak scale. At the scale $m_{b}$ the operators in Eq. (49) can be matched onto operators in the SCET. The four linearly independent gauge invariant operators are [3, 14]

$$
\begin{aligned}
& Q_{\mathbf{0}}^{1,5}=\left(\bar{c}_{v^{\prime}} \Gamma_{h}^{1,5} b_{v}\right)\left(\bar{\xi}_{n, p^{\prime}}^{(d)} W C_{\mathbf{0}}^{1,5}\left(\overline{\mathcal{P}}, \overline{\mathcal{P}}^{\dagger}\right) \Gamma_{\ell} W^{\dagger} \xi_{n, p}^{(u)}\right) \\
& Q_{\mathbf{8}}^{1,5}=\left(\bar{c}_{v^{\prime}} S T^{a} S^{\dagger} \Gamma_{h}^{1,5} b_{v}\right)\left(\bar{\xi}_{n, p^{\prime}}^{(d)} W C_{\mathbf{8}}^{1,5}\left(\overline{\mathcal{P}}, \overline{\mathcal{P}}^{\dagger}\right) T^{a} \Gamma_{\ell} W^{\dagger} \xi_{n, p}^{(u)}\right),
\end{aligned}
$$

where $\Gamma_{h}^{1,5}=\not h / 2, \not h \gamma_{5} / 2$ and $\Gamma_{\ell}=\not h P_{L} / 2$. Note that the hard Wilson coefficients $C_{\mathbf{0}, \mathbf{8}}^{j}\left(\overline{\mathcal{P}}, \overline{\mathcal{P}}^{\dagger}\right)$ are functions of the label operator and appear between gauge invariant combinations of collinear fields.

In Eq. (50) $c_{v^{\prime}}$ and $b_{v}$ are the usual HQET fields with the Lagrangian in Eq. (30). For the color singlet operators $Q_{0}^{1,5}$ the product $\bar{c}_{v^{\prime}} \Gamma b_{v}$ is invariant under soft gauge transformations and factors of $S$ do not appear. Equivalently, if we integrate out offshell fluctuations induced by coupling the soft gluons and collinear particles as in Appendix A, then we obtain 


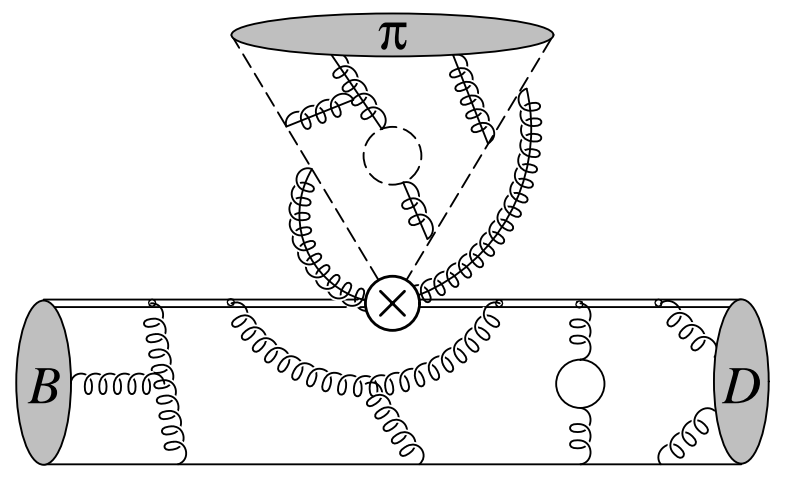

FIG. 7. Example illustrating how the soft and collinear gluons factor in the $B \rightarrow D \pi$ matrix element in the soft-collinear effective theory. The $\otimes$ denotes an insertion of $Q_{\mathbf{0}}^{1}$, the double lines are for $b_{v}$ or $c_{v^{\prime}}$, the springs with a line are collinear gluons, the dashed lines are collinear quarks, the springs without a line are soft gluons, and the normal solid lines are soft quarks.

$\bar{\xi}_{n, p^{\prime}} W S^{\dagger} C_{\mathbf{0}} S W^{\dagger} \xi_{n, p}$ and the soft gluon couplings cancel since they commute with $C_{\mathbf{0}}$ and $S^{\dagger} S=1$. Thus, no soft gluons appear in the color singlet case and the matrix element of the effective theory operator factors. In the color octet case $\bar{c}_{v^{\prime}} T^{a} b_{v}$ is not gauge invariant, but invariance of $S^{\dagger} b_{v}$ and $\bar{c}_{v^{\prime}} S$ imply that $\bar{c}_{v^{\prime}} S T^{a} S^{\dagger} b_{v}$ and hence $Q_{8}^{1,5}$ are gauge invariant. In this case factors of $S$ appear in the operator, but do so in a way that preserves the color octet structure of the matrix between the heavy quarks. Therefore, the effective theory matrix element of $Q_{8}^{1,5}$ is zero between physical color singlet states.

For completeness we note that the coupling of all usoft gluons to collinear fields in the $B \rightarrow D \pi$ matrix element also factor. Following Sec. III.A we redefine the collinear fields by $\xi_{n, p}=Y \xi_{n, p}^{(0)}$ and $W=Y W^{(0)} Y^{\dagger}$. For the color-singlet operator the identity $Y Y^{\dagger}=1$, together with the cancellation of the usoft couplings in the collinear Lagrangian, implies that the usoft gluons factor from the collinear part of the operator. For the color octet operator the same conclusion follows once we use the color identity $T^{a} \otimes Y^{\dagger} T^{a} Y=Y T^{a} Y^{\dagger} \otimes T^{a}$. This identity is easily derived by noting that $Y T^{a} Y^{\dagger}=\mathcal{Y}^{b a} T^{b}$ and using the properties of $Y$ in the adjoint representation.

Since the (u)soft and collinear particles decouple in $Q_{0}^{1,5}$ the matrix element for $B \rightarrow D \pi$ factors into a soft matrix element for $B \rightarrow D$ and a collinear matrix element for vacuum to $\pi$. In Ref. [14] it was also shown that the dependence of the Wilson coefficients $C_{\mathbf{0}}^{1,5}\left(\mu, \overline{\mathcal{P}}_{+}\right)$ on $\overline{\mathcal{P}}_{+}=\overline{\mathcal{P}}+\overline{\mathcal{P}}^{\dagger}$ leads to a non-trivial convolution of this hard coefficient with the light-cone pion wavefunction described by the matrix element of collinear fields. The only gluons giving non-canceling contributions to the $B \rightarrow D \pi$ matrix element appear as in the example in Fig. 0. This resulted in the first proof [14] of the $B \rightarrow D \pi$ factorization formula (proposed in Refs. [12,13])

\footnotetext{
${ }^{8}$ For soft heavy quarks this proof is not really necessary, since the coupling of an usoft gluon to a soft heavy quark is order $\lambda$, ie. power suppressed.
} 


$$
\left\langle D_{v^{\prime}} \pi_{n}\left|Q_{\mathbf{0}}^{1}\right| B_{v}\right\rangle=N F^{B \rightarrow D}(0) \int_{0}^{1} d x T(x, \mu) \phi_{\pi}(x, \mu)
$$

to all orders in $\alpha_{s}$ and leading order in $\Lambda_{\mathrm{QCD}} / Q$ where $Q=m_{b}, m_{c}$, or $E_{\pi}$. Here $N=$ $i_{B} E_{\pi} f_{\pi} / 2, F^{B \rightarrow D}\left(q^{2}\right)$ is the soft $B \rightarrow D$ form factor (Isgur-Wise function), $T(x, \mu)=$ $C_{\mathbf{0}}^{1}\left(\mu,(4 x-2) E_{\pi}\right)$ is the hard Wilson coefficient, and $\phi_{\pi}(x, \mu)$ is the nonperturbative lightcone pion wavefunction determined by a matrix element of collinear fields.

\section{B. Factorization in inclusive $B \rightarrow X_{s} \gamma$ decays}

The weak radiative decay $B \rightarrow X_{s} \gamma$ is mediated by the effective Hamiltonian

$$
\mathcal{H}=-\frac{4 G_{F}}{\sqrt{2}} V_{t b} V_{t s}^{*} C_{7} \mathcal{O}_{7}, \quad \mathcal{O}_{7}=\frac{e}{16 \pi^{2}} m_{b} \bar{s} \sigma_{\mu \nu} F^{\mu \nu} P_{R} b
$$

with $F_{\mu \nu}$ the electromagnetic field tensor and $P_{R}=\frac{1}{2}\left(1+\gamma_{5}\right)$. (The contributions from operators other than $\mathcal{O}_{7}$ are neglected here.) We define the kinematics of the decay such that the photon momentum $q$ is along the light cone $\bar{n}$ direction, $q_{\mu}=E_{\gamma} \bar{n}_{\mu}$. Here $E_{\gamma}=v \cdot q$ is the photon energy in the rest frame of the $B$ meson $\left(p_{B}=m_{B} v\right)$.

The inclusive photon energy spectrum can be written using the optical theorem as

$$
\frac{1}{\Gamma_{0}} \frac{d \Gamma}{d E_{\gamma}}=\frac{4 E_{\gamma}}{m_{b}^{3}}\left(-\frac{1}{\pi}\right) \operatorname{Im} T\left(E_{\gamma}\right),
$$

where the forward scattering amplitude $T\left(E_{\gamma}\right)$ is

$$
T\left(E_{\gamma}\right)=\frac{i}{m_{B}} \int d^{4} x e^{-i q \cdot x}\left\langle\bar{B}\left|T J_{\mu}^{\dagger}(x) J^{\mu}(0)\right| \bar{B}\right\rangle,
$$

with relativistic normalization for the $|\bar{B}\rangle$ states. Here the current $J_{\mu}=\bar{s} i \sigma_{\mu \nu} q^{\nu} P_{R} b$, and

$$
\Gamma_{0}=\frac{G_{F}^{2} m_{b}^{5}}{32 \pi^{4}}\left|V_{t b} V_{t s}^{*}\right|^{2} \alpha_{\mathrm{em}}\left|C_{7}^{F}\left(m_{b}\right)\right|^{2}
$$

is the parton level decay rate for $b \rightarrow s \gamma$ with the Wilson coefficient $C_{7}^{F}$ obtained by running down from the weak scale.

In the endpoint region $m_{B} / 2-E_{\gamma} \lesssim \Lambda_{\mathrm{QCD}}$ the spectrum can not be described by a completely local operator product expansion. However, it can be described by a twist expansion. For this region of phase space the time ordered product in Eq. (54) becomes simpler once we match onto effective theory fields and drop power corrections. At leading order in $\lambda$ we will show that $d \Gamma / d E_{\gamma}$ can be written in the factorized form [0]

$$
\frac{1}{\Gamma_{0}} \frac{d \Gamma}{d E_{\gamma}}=H\left(m_{b}, \mu\right) \int_{2 E_{\gamma}-m_{b}}^{\bar{\Lambda}} d k^{+} S\left(k^{+}, \mu\right) J\left(k^{+}+m_{b}-2 E_{\gamma}, \mu\right) .
$$

The different factors account for the contributions of different distance scales, and their $\mu$ dependence cancels. The factor $H\left(m_{b}, \mu\right)$ arises from hard gluons and is calculable perturbatively as an expansion in $\alpha_{s}\left(m_{b}\right)$. The jet factor $J$ contains the contributions from the 
collinear particles, while the usoft matrix element $S$ describes the nonperturbative dynamics of the usoft modes.

We start by matching the weak current onto an operator in the soft-collinear effective theory. At leading order in $\lambda$ this gives

$$
J_{\mu}=-E_{\gamma} e^{i\left(\overline{\mathcal{P}} \frac{n}{2}+\mathcal{P}_{\perp}-m_{b} v\right) \cdot x}\left\{\left[2 C_{9}(\overline{\mathcal{P}}, \mu)+C_{12}(\overline{\mathcal{P}}, \mu)\right] J_{\mu}^{\text {eff }}-C_{10}(\overline{\mathcal{P}}, \mu) \widetilde{J}_{\mu}^{\text {eff }}\right\},
$$

where

$$
J_{\mu}^{\mathrm{eff}}=\bar{\xi}_{n, p} W \gamma_{\mu}^{\perp} P_{L} b_{v}, \quad \widetilde{J}_{\mu}^{\mathrm{eff}}=\bar{n}_{\mu} \bar{\xi}_{n, p} W P_{R} b_{v}
$$

The SCET Wilson coefficients $C_{9,10,12}(\overline{\mathcal{P}}, \mu)$ are given at one-loop in Eq. (33) of Ref. [2]. The $x$ dependence of the currents will lead to conservation of both label and residual momenta separately. For example, for label momenta $p, p^{\prime}$ and residual momenta $k, k^{\prime}$

$$
\int d^{4} x e^{i\left(p-p^{\prime}+k-k^{\prime}\right) \cdot x}=\delta_{p, p^{\prime}}(2 \pi)^{4} \delta^{4}\left(k-k^{\prime}\right)=\delta_{p, p^{\prime}} \int d^{4} x e^{i\left(k-k^{\prime}\right) \cdot x} .
$$

In Eq. (57) label conservation sets $\overline{\mathcal{P}}=m_{b}$ and $\mathcal{P}_{\perp}=0$ and the remaining momenta in the time-ordered product will be purely residual. The current $\tilde{J}_{\mu}^{\text {eff }}$ does not contribute for real transversely polarized photons, and will be omitted from the future discussion. Inserting Eq. (57) into Eq. (54) we can write to leading order

$$
\frac{4 E_{\gamma}}{m_{b}^{3}} T\left(E_{\gamma}\right) \equiv H\left(m_{b}, \mu\right) T^{\mathrm{eff}}\left(E_{\gamma}, \mu\right)
$$

Here $T^{\mathrm{eff}}$ is the forward scattering amplitude in the effective theory

$$
T^{\mathrm{eff}}=i \int d^{4} x e^{i\left(m_{b} \frac{\bar{n}}{2}-q\right) \cdot x}\left\langle\bar{B}_{v}\left|\mathrm{~T} J_{\mu}^{\mathrm{eff} \dagger}(x) J^{\mathrm{eff} \mu}(0)\right| \bar{B}_{v}\right\rangle,
$$

with HQET normalization for the states [16], and in terms of the SCET Wilson coefficients the hard amplitude is

$$
H\left(m_{b}, \mu\right)=\frac{16 E_{\gamma}^{3}}{m_{b}^{3}}\left|C_{9}\left(m_{b}, \mu\right)+\frac{1}{2} C_{12}\left(m_{b}, \mu\right)\right|^{2} .
$$

Next the usoft gluons in $T^{\mathrm{eff}}$ can be decoupled from the collinear fields as explained in Sec. III, by making the substitutions

$$
\xi_{n, p} \rightarrow Y \xi_{n, p}^{(0)}, \quad W \rightarrow Y W^{(0)} Y^{\dagger}
$$

This results in

$$
J_{\mu}^{\mathrm{eff}}=\bar{\xi}_{n, p}^{(0)} W^{(0)} \gamma_{\mu}^{\perp} P_{L} Y^{\dagger} b_{v}
$$

where the collinear fields in this current do not interact with usoft fields. With this current an example of the type of SCET graph contributing to $T^{\mathrm{eff}}$ is shown in Fig. 8. Thus, the 


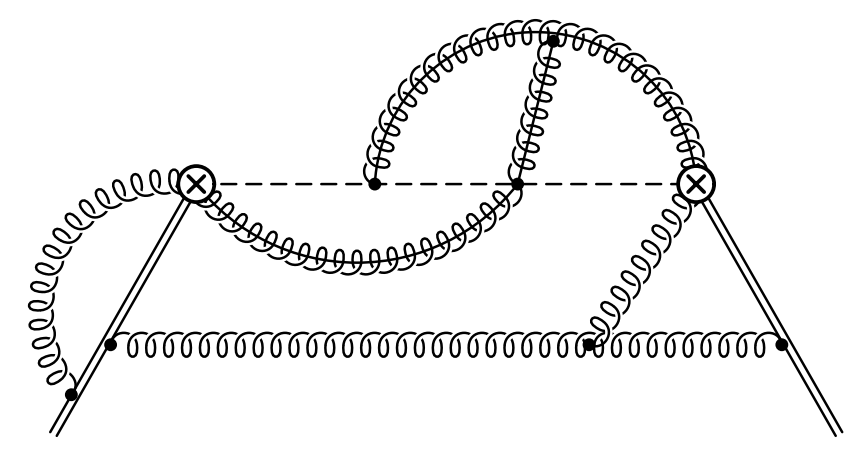

FIG. 8. Example of the type of graph that contributes to $B \rightarrow X_{s} \gamma$ in the soft-collinear effective theory. The $\otimes$ denotes an insertion of the current in Eq. (64), the double lines are $b_{v}$ quarks, the springs with a line are collinear gluons, and the springs without a line are usoft gluons.

time-ordered product of the effective theory currents is

$$
\begin{aligned}
T^{\mathrm{eff}} & =i \int d^{4} x e^{i\left(m_{b} \frac{\bar{n}}{2}-q\right) \cdot x}\left\langle\bar{B}_{v}\left|\mathrm{~T} J_{\mu}^{\mathrm{eff} \dagger}(x) J^{\mathrm{eff}, \mu}(0)\right| \bar{B}_{v}\right\rangle \\
& =i \int d^{4} x e^{i\left(m_{b} \frac{\bar{n}}{2}-q\right) \cdot x}\left\langle\bar{B}_{v}\left|\mathrm{~T}\left[\bar{b}_{v} Y P_{R} \gamma_{\mu}^{\perp} W^{(0) \dagger} \xi_{n, p}^{(0)}\right](x)\left[\bar{\xi}_{n, p}^{(0)} W^{(0)} \gamma_{\perp}^{\mu} P_{L} Y^{\dagger} b_{v}\right](0)\right| \bar{B}_{v}\right\rangle \\
& =-\int d^{4} x \int \frac{d^{4} k}{(2 \pi)^{4}} e^{i\left(m_{b} \frac{\bar{n}}{2}-q-k\right) \cdot x}\left\langle\bar{B}_{v}\left|\mathrm{~T}\left[\bar{b}_{v} Y\right](x) P_{R} \gamma_{\mu}^{\perp} \frac{\not h}{2} \gamma_{\perp}^{\mu} P_{L}\left[Y^{\dagger} b_{v}\right](0)\right| \bar{B}_{v}\right\rangle J_{P}(k) \\
& =\frac{1}{2} \int d^{4} x \int \frac{d^{4} k}{(2 \pi)^{4}} e^{i\left(m_{b} \frac{\bar{n}}{2}-q-k\right) \cdot x}\left\langle\bar{B}_{v}\left|\mathrm{~T}\left[\bar{b}_{v} Y\right](x)\left[Y^{\dagger} b_{v}\right](0)\right| \bar{B}_{v}\right\rangle J_{P}(k) .
\end{aligned}
$$

In the third line we used the fact that the $\bar{B}$ meson state contains no collinear particles, and we defined $J_{P}(k)$ as the Fourier transform of the contraction of all collinear fields

$$
\left\langle 0\left|\mathrm{~T}\left[W^{(0) \dagger} \xi_{n, p}^{(0)}\right](x)\left[\bar{\xi}_{n, p}^{(0)} W^{(0)}\right](0)\right| 0\right\rangle \equiv i \int \frac{d^{4} k}{(2 \pi)^{4}} e^{-i k \cdot x} J_{P}(k) \frac{\not h}{2},
$$

where the label $P$ equals the sum of the label momenta carried by the collinear fields in both $\left[W^{(0) \dagger} \xi_{n, p}^{(0)}\right]$ and $\left[\bar{\xi}_{n, p}^{(0)} W^{(0)}\right]$. In the fourth line of Eq. (65) the spin structure was simplified using the fact that $\psi b_{v}=b_{v}$, and that the $B$-meson is a pseudoscalar. To proceed further we can make use of the fact that $J_{P}$ only depends on the component $k^{+}=n \cdot k$ of the residual momentum $k$. This follows from the collinear Lagrangians in Eqs. (24) and (25) which contain only the $n \cdot \partial$ derivative. This simplification allows us to perform the $k_{-}, k_{\perp}$ integrations in Eq. (66) which puts $x$ on the light cone

$$
\begin{aligned}
T^{\mathrm{eff}} & =\frac{1}{2} \int d^{4} x e^{i\left(m_{b} \frac{\bar{n}}{2}-q\right) \cdot x} \delta\left(x^{+}\right) \delta\left(\vec{x}_{\perp}\right) \int \frac{d k_{+}}{2 \pi} e^{-\frac{i}{2} k_{+} x_{-}}\left\langle\bar{B}_{v}\left|\mathrm{~T}\left[\bar{b}_{v} Y\right](x)\left[Y^{\dagger} b_{v}\right](0)\right| \bar{B}_{v}\right\rangle J_{P}\left(k^{+}\right) \\
& =\frac{1}{2} \int d k^{+} J_{P}\left(k^{+}\right) \int \frac{d x^{-}}{4 \pi} e^{-\frac{i}{2}\left(2 E_{\gamma}-m_{b}+k^{+}\right) x^{-}}\left\langle\bar{B}_{v}\left|\mathrm{~T}\left[\bar{b}_{v} Y\right]\left(\frac{n}{2} x^{-}\right)\left[Y^{\dagger} b_{v}\right](0)\right| \bar{B}_{v}\right\rangle .
\end{aligned}
$$

\footnotetext{
${ }^{9}$ Even though the usoft and collinear fields factor in the current the same $\mu$ must be used when renormalizing loops involving usoft or collinear gluons.
} 


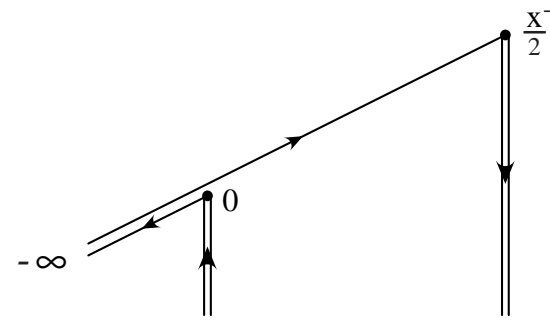

FIG. 9. Wilson lines that appear in the time-ordered product for $B \rightarrow X_{s} \gamma$. The vertical double lines represent the heavy quarks along direction $v$ and the diagonal single lines are the usoft Wilson lines, $Y$, along direction $n$. The parts from $-\infty$ to 0 cancel leaving $Y(0, x)$.

Note that the typical offshellness of the collinear particles is $p^{2} \sim m_{b} \Lambda_{\mathrm{QCD}}$ so the function $J_{P}$ can be calculated perturbatively. At lowest order in $\alpha_{s}\left(\sqrt{m_{b} \Lambda_{\mathrm{QCD}}}\right), J_{P}\left(k^{+}\right)$is determined by the collinear quark propagator carrying momentum $(P+k)$

$$
J_{P}\left(k^{+}\right)=\frac{\bar{n} \cdot P}{(P+k)^{2}+i \epsilon}=\frac{1}{n \cdot k+P_{\perp}^{2} /(\bar{n} \cdot P)+i \epsilon} .
$$

Finally, the remaining matrix element in Eq. (67) is purely usoft

$$
\begin{aligned}
S\left(l^{+}\right) & \equiv \frac{1}{2} \int \frac{d x^{-}}{4 \pi} e^{-\frac{i}{2} l^{+} x^{-}}\left\langle\bar{B}_{v}\left|T\left[\bar{b}_{v} Y\right]\left(\frac{n}{2} x^{-}\right)\left[Y^{\dagger} b_{v}\right](0)\right| \bar{B}_{v}\right\rangle \\
& =\frac{1}{2} \int \frac{d x^{-}}{4 \pi} e^{-\frac{i}{2} l^{+} x^{-}}\left\langle\bar{B}_{v}\left|T \bar{b}_{v}\left(\frac{n}{2} x^{-}\right) P \exp \left(i g \int_{0}^{x^{-} / 2} d \lambda n \cdot A(n \lambda)\right) b_{v}(0)\right| \bar{B}_{v}\right\rangle .
\end{aligned}
$$

In the second line we have used the multiplicative nature of the Wilson lines $Y\left(\frac{x^{-}}{2}\right) Y^{\dagger}(0)$ illustrated in Fig. 9. If we use $h_{v}^{(0)}$ and $S_{v}$ from section II then Eq. (67) reproduces the Wilson line contour in Ref. [7]. The universal nonperturbative function $S\left(k^{+}\right)$encodes all the relevant information about the usoft dynamics of the $B$ meson, and is the structure function introduced in Ref. [6]

$$
S\left(k^{+}\right)=\frac{1}{2}\left\langle\bar{B}_{v}\left|\bar{b}_{v} \delta\left(i n \cdot D-k^{+}\right) b_{v}\right| \bar{B}_{v}\right\rangle
$$

where the $1 / 2$ accounts for our normalization for the states. Eq. (70) makes clear the physical interpretation of $S\left(k^{+}\right)$as the probability to find the $b$ quark inside the $\bar{B}$ meson carrying a residual momentum of light-cone component $k^{+}$. The structure function is real, has support over the infinite range $-\infty \leq k^{+} \leq \bar{\Lambda}$, and peaks around $k^{+} \simeq 0$.

Inserting Eq. (69) into Eq. (67) and taking the imaginary part gives

$$
\frac{1}{\Gamma_{0}} \frac{d \Gamma}{d E_{\gamma}}=H\left(m_{b}, \mu\right) \int_{2 E_{\gamma}-m_{b}}^{\bar{\Lambda}} d k^{+} S\left(k^{+}\right) J\left(k^{+}+m_{b}-2 E_{\gamma}\right) .
$$

with the jet function

$$
J\left(k^{+}\right) \equiv-\frac{1}{\pi} \operatorname{Im} J_{P}\left(k^{+}\right) .
$$


The result in Eq. (71) agrees with Ref. [7] and is valid to all orders in $\alpha_{s}$ and leading order in $\Lambda_{\mathrm{QCD}} / Q$ where $Q=E_{\gamma}$ or $m_{b}$. The lower limit of integration is fixed by the fact that $J\left(k^{+}\right)$is nonzero only for positive values of its argument, and the upper limit is fixed by the support of the shape function $S\left(k^{+}\right)$. In summary, at leading order in $\lambda$ the photon energy spectrum is given by the hard coefficient in Eq. (62) and collinear function $J\left(k^{+}\right)$ both calculable perturbatively, together with the nonperturbative structure function $S\left(k^{+}\right)$ of the $B$ meson. Thus, Eq. (71) shows how to match onto this shape function consistently at any order in $\alpha_{s}$.

\section{CONCLUSIONS}

In this paper we considered the interactions of collinear, soft, and ultrasoft (usoft) particles in an effective theory. The soft-collinear effective theory (SCET) is organized with a power counting in $\lambda$ or equivalently $\Lambda_{\mathrm{QCD}} / Q$, where $Q$ denotes the large momentum in an energetic process or the large mass of a heavy quark. The lowest order Lagrangian is determined by power counting together with collinear, soft, and usoft gauge invariance. Collinear gauge invariance acts like a reparameterization invariance on collinear fields, and constrains Wilson coefficients to only depend on the large momentum picked out by the label operator $\overline{\mathcal{P}}$.

In the collinear Lagrangian the usoft gluons appear as background fields. Power counting and gauge invariance allow only the $n \cdot \partial$ usoft momentum and $n \cdot A_{u s}$ usoft gluons to appear in the collinear Lagrangian at leading order in $\lambda$. At this order a field redefinition involving a Wilson line $Y$ was identified under which all usoft gluons are decoupled from collinear fields, while reappearing explicitly in collinear operators. Soft gluons couple in a somewhat different manner. Since they can not interact with a collinear particle without taking it far offshell they do not show up in the collinear Lagrangian. A collinear-soft interaction produces a particle with momenta $Q(\lambda, 1, \lambda)$, and these are integrated out in constructing operators in the effective theory. This was done to all orders in Appendix A. As a result the $n \cdot A_{s}$ soft gluons appear in a Wilson line $S$ which shows up in a way that preserves the gauge invariance of operators with soft and collinear fields. This is similar to the appearance of the collinear Wilson line $W$ which is necessary to construct gauge invariant operators with collinear fields [3]. With the $n \cdot A$ usoft and soft gluons explicit in operators, the manner in which they factor from collinear fields is readily seen.

Two examples of the simplicity of factorization in the effective theory were given. As an exclusive example we discussed $B \rightarrow D \pi$ in section $\nabla A$. In the limit of infinitely heavy quarks and pion energy we discussed how soft and usoft gluons decouple from the collinear quarks and gluons that make up the pion. In section $\mathrm{VB}$ we discussed factorization for the inclusive decay $B \rightarrow X_{s} \gamma$ in the region of large photon energy. In this case usoft gluons factor from the $X_{s}$ collinear jet function, $J\left(k^{+}\right)$, in a way that leaves a nontrivial convolution of $J\left(k^{+}\right)$with a usoft light cone structure function, $S\left(k^{+}\right)$, for the $B$ meson. 
Throughout this paper we have focused on results which appear at leading order in $\lambda$ in the SCET. However, the real advantage of the effective theory approach is that the structure of power corrections can be addressed in a systematic way. To do so one must simply extend the effective Lagrangian and currents to subleading orders in $\lambda$. In the language we have developed such an analysis should be quite similar to the analysis of $1 / m$ corrections in HQET. In general the resulting $\mathcal{O}(\lambda)$ results will be described in terms of the same $(\mathrm{u})$ soft and collinear degrees of freedom, but will not necessarily obey factorization formulae. The power of the effective theory language is that it is general enough to describe these corrections in terms of subleading operators.

\section{ACKNOWLEDGMENTS}

This work was supported by the DOE under grant DOE-FG03-97ER40546 and by NSERC of Canada. We would like to thank the Aspen Center for Physics for providing a stimulating environment while this paper was completed. We would also like to thank I. Rothstein and S. Fleming for helpful discussions. 


\section{APPENDIX A: INTEGRATING OUT $Q(\lambda, 1, \lambda)$ MODES}

In this Appendix we explicitly integrate out the offshell modes that arise in QCD when soft and collinear particles couple to one another. In section IVB an order $g^{2}$ matching calculation was performed in which offshell modes were integrated out leaving behind the collinear and a soft Wilson lines $W$ and $S$. This calculation was performed by matching directly from QCD onto the effective theory, without specifically identifying the offshell fluctuations. Since the sum of a soft and collinear momenta gives $p \sim Q(\lambda, 1, \lambda)$ some offshell propagators have offshellness $p^{2} \sim Q^{2} \lambda \ll Q^{2}$, and it is interesting to see how they can be integrated out of the theory reliably to all orders in the coupling.

To facilitate integrating out the offshell modes to all orders we find it useful to introduce auxiliary fields for these fluctuations as an intermediate step. First we match onto a Lagrangian with couplings between the onshell and offshell fields, and then the offshell fields are explicitly integrated out. A simple example illustrating how this works is the appearance of $W$ in the current coupling an ultrasoft heavy quark and a collinear quark, $\bar{\xi}_{n, p} W \Gamma h_{v}$. The full QCD calculation was displayed in Fig. 1. Instead of immediately integrating out the offshell lines, consider first matching onto an action with an auxiliary field $\psi_{H}$ for the offshell heavy quark. The vertices then include the initial production of the $\psi_{H}$, its interaction with the $\bar{n} \cdot A_{n, q}$ gluons, and its annihilation at the current. For the auxiliary Lagrangian and current we find

$$
\mathcal{L}_{\text {aux }}\left[\psi_{H}\right]=\bar{\psi}_{H} g \bar{n} \cdot A_{n, q} h_{v}+\bar{\psi}_{H}\left(\bar{n} \cdot \mathcal{P}+g \bar{n} \cdot A_{n, q}\right) \psi_{H}, \quad J=\bar{\xi}_{n, p} \Gamma\left(h_{v}+\psi_{H}\right)
$$

The spin structure in the vertices and propagator always multiply to give a projector on the final onshell field $\psi h_{v}=h_{v}$, so we have simplified $\mathcal{L}_{\text {aux }}$ by suppressing this structure. We also will suppress the $\bar{n} \cdot p$ label on the field $\psi_{H}$. Unlike for onshell fields, the power counting for $\psi_{H}$ is not unique. Choosing the measure $d^{4} x \sim \lambda^{-2 b}$ one finds $\psi_{H} \sim \lambda^{b}$. However, this arbitrary $b$ dependence cancels between the vertices in which $\psi_{H}$ is produced and the current which annihilates $\psi_{H}$. This makes any graph involving the vertices in Eq. (A1) order $\lambda^{0}$. Using Eq. (11) one can solve the equation of motion for the $\psi_{H}$ field in terms of the usoft heavy quark field $h_{v}$,

$$
\psi_{H}=(W-1) h_{v} .
$$

This solution sums the tree level graphs in Fig. 1. Inserting Eq. (A2) into $J$ in Eq. (A1) then gives

$$
J=\bar{\xi}_{n, p} W \Gamma h_{v}
$$

which is the expected gauge invariant current in the effective theory. This is the same result obtained in Ref. [2] by explicit matching from QCD.

For the case of offshell fluctuations induced by soft-collinear interactions the situation is more complicated and the above auxiliary field approach turns out to be crucial for an 


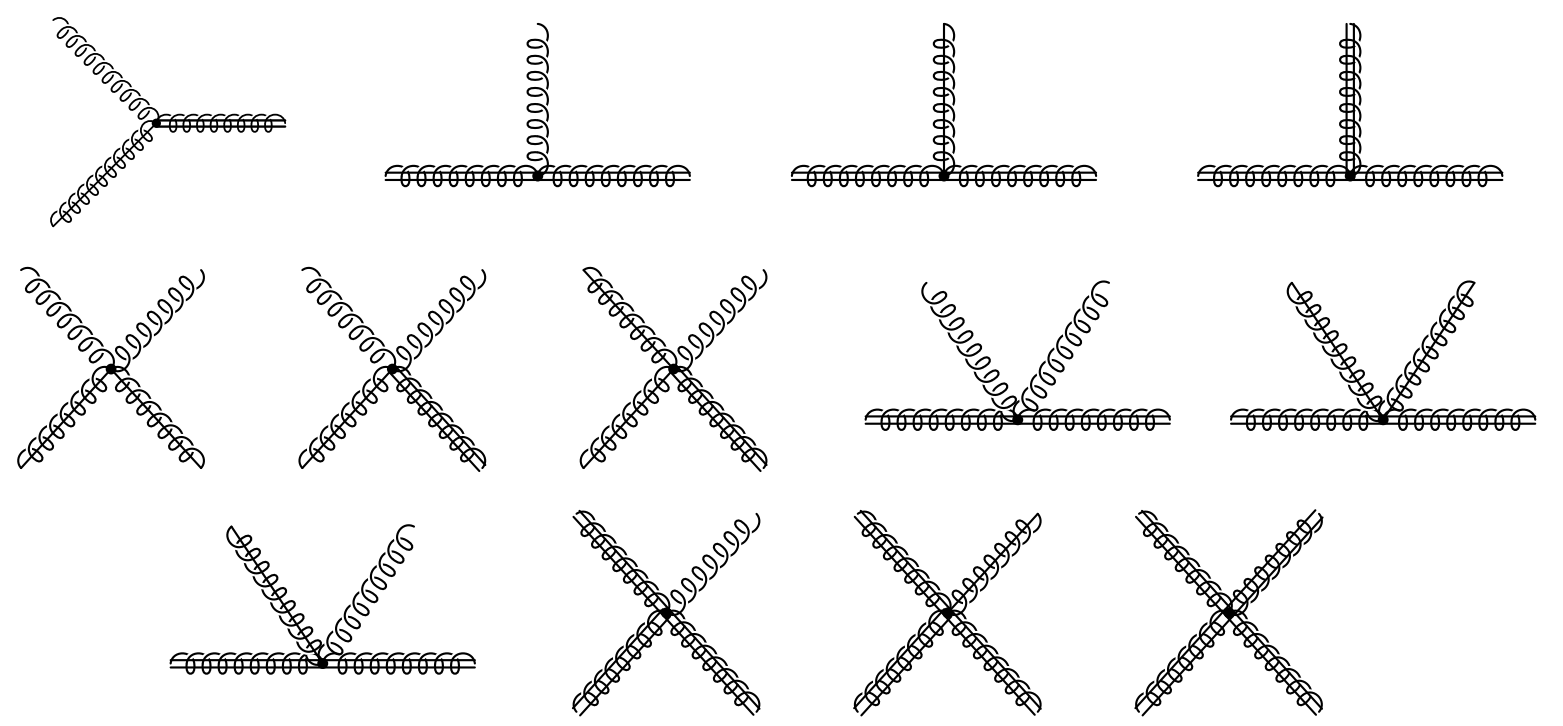

FIG. 10. Mixed gluon vertices with offshell $A_{X}^{\mu}$ gluons (spring with a double line), soft gluons (spring), and collinear gluons (spring with a single line). Purely soft or purely collinear vertices are not shown.

all orders matching calculation. In this case collinear gluons still knock the soft particles offshell, but the soft particles also knock the collinear particles offshell. Therefore, one needs auxiliary fields $\psi_{H}, \psi_{L}$, and $A_{X}^{\mu}$ for the offshell heavy quarks, offshell collinear quarks, and offshell gluons, respectively. Here $\psi_{H}$ is offshell by $p^{2} \sim Q^{2}$, while $\psi_{L}$ and $A_{X}^{\mu}$ are offshell by $p^{2} \sim Q^{2} \lambda$. Both of these scales are much greater than the fluctuation scales for onshell particles which have $p^{2} \lesssim Q^{2} \lambda^{2}$. For simplicity we will suppress both the $n \cdot p \sim \lambda$ labels on $\psi_{L}$ and $A_{X}^{\mu}$ and the $\bar{n} \cdot p \sim \lambda^{0}$ label on $A_{X}^{\mu}$. Just like $\psi_{H}$, the power counting for the $\psi_{L}$ and $A_{X}^{\mu}$ fields is not unique, but this dependence again cancels between production and annihilation vertices in any graph. Taking $d^{4} x \sim \lambda^{-2 \ell}$ one finds $\psi_{L} \sim \lambda^{\ell-1 / 2}$ and $\left(n \cdot A_{X}, \bar{n} \cdot A_{X}, A_{X}^{\perp}\right) \sim\left(\lambda^{\ell}, \lambda^{\ell-1}, \lambda^{\ell-1 / 2}\right)$.

To ensure gauge invariance under soft and collinear gauge transformations, the gluon fields $A_{s, p}^{\mu}$ and $A_{n, q}^{\mu}$ are included as background fields. We also include interactions with a soft quark, $q_{s}$, and a collinear quark, $\xi_{n, p}$. The Lagrangian for the interaction of these fields with the offshell modes can be obtained by expanding the QCD Feynman rules and in each case keeping only the leading term in $\lambda$. From these Feynman rules one can construct the quark and gluon Lagrangians for interactions with the auxiliary fields, $\psi_{H}, \psi_{L}, A_{X}$. For the auxiliary quark Lagrangian we obtain

$$
\begin{aligned}
\mathcal{L}_{\text {aux }}^{(q)}\left[\psi_{H}, \psi_{L}, A_{X}\right]= & \bar{\psi}_{H}\left(g \bar{n} \cdot A_{X}+g \bar{n} \cdot A_{n, q}\right) h_{v}+\bar{\psi}_{H}\left(\overline{\mathcal{P}}+g \bar{n} \cdot A_{X}+g \bar{n} \cdot A_{n, q}\right) \psi_{H} \\
& +\bar{\xi}_{n, p}\left(g n \cdot A_{X}+g n \cdot A_{s, p}\right) \psi_{L}+\bar{\psi}_{L}\left(n \cdot \mathcal{P}+g n \cdot A_{X}+g n \cdot A_{s, p}\right) \psi_{L},
\end{aligned}
$$

where again the spin structure is suppressed. We see explicitly that only the $\bar{n} \cdot A_{n, q}$ and $n \cdot A_{s}$ components appear. This is also true for pure gluon vertices, and expanding we find contributions from the graphs shown in Fig. 10. The Feynman rules for these graphs are reproduced by the auxiliary gluon Lagrangian 


$$
\mathcal{L}_{\text {aux }}^{(g)}\left[A_{X}\right]=\frac{1}{2 g^{2}} \operatorname{tr}\left\{\left[i D_{X}^{\mu}+g A_{X}^{\mu}, i D_{X}^{\nu}+g A_{X}^{\nu}\right]\right\}^{2}+\frac{1}{\alpha_{L}} \operatorname{tr}\left\{\left[i D_{X \mu}, A_{X}^{\mu}\right]\right\}^{2}
$$

where

$$
i D_{X}^{\mu}=\frac{n^{\mu}}{2}\left(\overline{\mathcal{P}}+g \bar{n} \cdot A_{n, q}\right)+\frac{\bar{n}^{\mu}}{2}\left(n \cdot \mathcal{P}+g n \cdot A_{s, p}\right) .
$$

Recall that $\overline{\mathcal{P}}$ picks out only the $\bar{n} \cdot p$ component of the momentum label which is order $\lambda^{0}$ and $n \cdot \mathcal{P}$ picks out the $n \cdot p$ label of order $\lambda$. The terms in $\mathcal{L}_{\text {aux }}^{(g, q)}$ do not scale homogeneously with $\lambda$, but all graphs with auxiliary fields on only internal lines are order $\lambda^{0}$. Finally, we note that $\mathcal{L}_{\text {aux }}^{(g)}$ is symmetric under the interchanges

$$
\bar{n} \leftrightarrow n, \quad \overline{\mathcal{P}} \leftrightarrow n \cdot \mathcal{P}, \quad \bar{n} \cdot A_{n, q} \leftrightarrow n \cdot A_{s, p}
$$

A further simplification can be achieved by taking

$$
A_{X}^{\mu}=\frac{n^{\mu}}{2} \bar{n} \cdot A_{X}+\frac{\bar{n}^{\mu}}{2} n \cdot A_{X}
$$

This is sufficient since all the $A_{X}^{\perp}$ gluons come in pairs, and can therefore never be produced and subsequently annihilated by coupling to the external $\bar{n} \cdot A_{n, q}$ and $n \cdot A_{s, p}$ fields. Finally, since the auxiliary fields are far offshell and don't really propagate, loops involving $\psi_{H}$, $\psi_{L}$, or $A_{X}^{\mu}$ (or offshell ghosts) do not need to be considered. (Such loops could contribute to hard corrections, but can not spoil the infrared structure of the operator generated by eliminating these modes.) Thus, for our purposes Eqs. (A4) and (A5) reduce to a classical two dimensional QCD action coupled to external adjoint and fundamental sources.

We begin by integrating out $\psi_{H}$ and $\psi_{L}$, and find solutions similar to the result in Eq. (A2)

$$
\psi_{H}=\left(W_{X}-1\right) h_{v}, \quad \psi_{L}=\left(S_{X}-1\right) \xi_{n, p},
$$

Here $W_{X}$ and $S_{X}$ satisfy

$$
\left(\overline{\mathcal{P}}+\bar{n} \cdot A_{X}+\bar{n} \cdot A_{n, q}\right) W_{X}=0, \quad\left(n \cdot \mathcal{P}+n \cdot A_{X}+n \cdot A_{s, p}\right) S_{X}=0,
$$

and are essentially the Fourier transforms of the Wilson lines

$$
\begin{aligned}
& W_{X}(y)=\mathrm{P} \exp \left\{i g \int_{-\infty}^{y} d s\left[\bar{n} \cdot A_{X}(s \bar{n})+\bar{n} \cdot A_{c}(s \bar{n})\right]\right\}, \\
& S_{X}(z)=\mathrm{P} \exp \left\{i g \int_{-\infty}^{z} d s\left[n \cdot A_{X}(s n)+n \cdot A_{s}(s n)\right]\right\},
\end{aligned}
$$

where $A_{c}$ and $A_{s}$ are the position space collinear and soft fields. The solutions in Eq. (A9) still contain the $n \cdot A_{X}$ and $\bar{n} \cdot A_{X}$ fields, which must be eliminated by solving the gluon Lagrangian in Eq. (A5).

The gluon action in Eq. (A5) contains two terms, $\mathcal{L}_{\text {aux }}^{(g 1)}+\frac{1}{\alpha_{L}} \mathcal{L}_{\text {aux }}^{(g 2)}$. The second is a gauge fixing term which removes the ambiguity associated with finding a definite solution for $A_{L}^{\mu}$. 
The two terms can be solved independently since $\alpha_{L}$ is arbitrary. We begin by solving the equations of motion for $\mathcal{L}_{\text {aux }}^{(g 1)}$ which are

$$
\left[i D_{X}^{\mu}+g A_{X}^{\mu},\left[i D_{X}^{\mu}+g A_{X}^{\mu}, i D_{X}^{\nu}+g A_{X}^{\nu}\right]\right]=0 .
$$

This is the direct analog of the QCD equations of motion $\left[D_{\mu}, F^{\mu \nu}\right]=0$. To proceed we write Eq. (A12) in terms of $W_{X}$ and $S_{X}$ using

$$
i D_{X}^{\mu}+g A_{X}^{\mu}=\frac{n^{\mu}}{2} W_{X} \overline{\mathcal{P}} W_{X}^{\dagger}+\frac{\bar{n}^{\mu}}{2} S_{X} n \cdot \mathcal{P} S_{X}^{\dagger} .
$$

to give

$$
\begin{aligned}
{\left[W_{X} \overline{\mathcal{P}} W_{X}^{\dagger},\left[S_{X} n \cdot \mathcal{P} S_{X}^{\dagger}, W_{X} \overline{\mathcal{P}} W_{X}^{\dagger}\right]\right] } & =0, \\
{\left[S_{X} n \cdot \mathcal{P} S_{X}^{\dagger},\left[W_{X} \overline{\mathcal{P}} W_{X}^{\dagger}, S_{X} n \cdot \mathcal{P} S_{X}^{\dagger}\right]\right] } & =0 .
\end{aligned}
$$

It is sufficient to only solve one of these equations since the second is equal to the first under the symmetry in Eq. (A7). Expanding the first equation and using $W_{X}^{\dagger} W_{X}=S_{X}^{\dagger} S_{X}=1$ gives

$$
2 W_{X} \overline{\mathcal{P}} W_{X}^{\dagger} S_{X}(n \cdot \mathcal{P}) S_{X}^{\dagger} W_{X} \overline{\mathcal{P}} W_{X}^{\dagger}-W_{X} \overline{\mathcal{P}}^{2} W_{X}^{\dagger} S_{X} n \cdot \mathcal{P} S_{X}^{\dagger}-S_{X} n \cdot \mathcal{P} S_{X}^{\dagger} W_{X} \overline{\mathcal{P}}^{2} W_{X}^{\dagger}=0 .
$$

We now make the following ansatz for a solution to this equation

$$
S_{X}^{\dagger} W_{X}=W S^{\dagger},
$$

which satisfies the symmetry in Eq. (A7). Inserting Eq. (A16) into Eq. (A15), and using $[\overline{\mathcal{P}}, S]=0$ and $[n \cdot \mathcal{P}, W]=0$ gives

$$
2 W_{X} S\left(\overline{\mathcal{P}}^{2} n \cdot \mathcal{P}\right) S^{\dagger} W_{X}^{\dagger}-W_{X} S\left(\overline{\mathcal{P}}^{2} n \cdot \mathcal{P}\right) W^{\dagger} S_{X}^{\dagger}-S_{X} W\left(\overline{\mathcal{P}}^{2} n \cdot \mathcal{P}\right) S^{\dagger} W_{X}^{\dagger} .
$$

Now the ansatz $S_{X}^{\dagger} W_{X}=W S^{\dagger}$ implies $W^{\dagger} S_{X}^{\dagger}=S^{\dagger} W_{X}^{\dagger}$ and $S_{X} W=W_{X} S$ so the three terms in Eq. (A17) cancel. Thus, Eq. (A16) is indeed a solution of the equations of motion for $\mathcal{L}_{\text {aux }}^{(g 1)}$.

The solution in Eq. (A16) gives only one equation for the two unknowns, $\bar{n} \cdot A_{X}$ and $n \cdot A_{X}$. The remaining redundancy is removed by demanding the vanishing of the gauge fixing term

$$
2\left[i D_{X \mu}, A_{X}^{\mu}\right]=\left[\overline{\mathcal{P}}+g \bar{n} \cdot A_{n, q}, n \cdot A_{X}\right]+\left[n \cdot \mathcal{P}+g n \cdot A_{s, p}, \bar{n} \cdot A_{X}\right]=0 .
$$

In terms of $W_{X}$ and $S_{X}$ Eq. (A18) implies that

$$
\left[W \overline{\mathcal{P}} W^{\dagger}, S_{X} n \cdot \mathcal{P} S_{X}^{\dagger}\right]+\left[S n \cdot \mathcal{P} S^{\dagger}, W_{X} \overline{\mathcal{P}} W_{X}^{\dagger}\right]=0 .
$$

Together Eqs. (A9), (A16), and (A18) solve the Lagrangian for the auxiliary quark and gluon fields and sum up all graphs with offshell lines that involve heavy and light fermions coupling to soft and collinear gluons. 


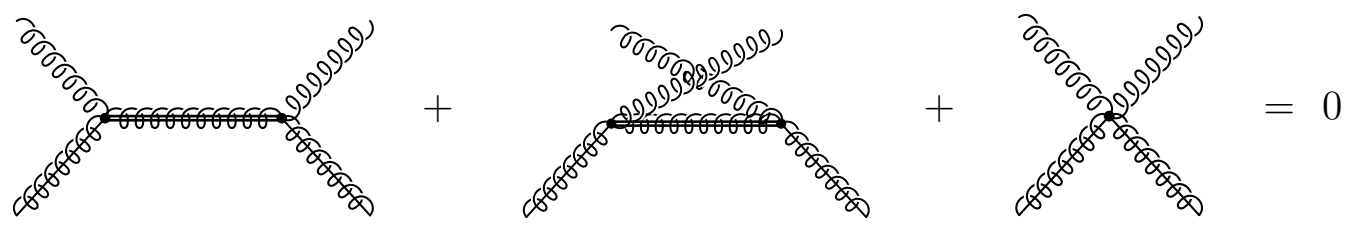

FIG. 11. Example of pure glue graphs which look like they could induce a four gluon soft-soft-collinear-collinear coupling, but add up to zero.

It should be emphasized that the Lagrangian in Eq. (A5) does not induce pure glue operators which couple soft and collinear gluons. Using Eq. (A13) to write $\mathcal{L}_{\text {aux }}^{(g 1)}$ in terms of $W_{X}$ and $S_{X}$, and then substituting in the solution in Eq. (A16) gives $\mathcal{L}_{\text {aux }}^{(g 1)}=0$ (using similar techniques to those used for the equations of motion). Furthermore, Eq. (A18) implies that $\mathcal{L}_{\text {aux }}^{(g 2)}=0$. Thus, no pure glue soft-collinear couplings are induced by integrating out the $\bar{n} \cdot A_{X}$ and $n \cdot A_{X}$ fields. This fact can also be seen perturbatively. As an example consider the diagrams in in Fig. 11. Here we show the three graphs with vertices from Eq. (A5) that contribute to the four point function with two soft and two collinear gluons. Adding the three graphs gives zero, so no operator with two soft and two collinear gluons is induced.

As a nontrivial example of the above results consider the heavy-to-light soft-collinear current discussed in section IVB. In terms of the auxiliary fields this current is

$$
J=\left(\bar{\psi}_{L}+\bar{\xi}_{n, p}\right) \Gamma\left(h_{v}+\psi_{H}\right) .
$$

Inserting into $J$ the result in Eq. (A9) we find

$$
J=\bar{\xi}_{n, p} S_{X}^{\dagger} \Gamma W_{X} h_{v} .
$$

Finally using Eq. (A16) gives

$$
J=\bar{\xi}_{n, p} W \Gamma S^{\dagger} h_{v}
$$

Thus, integrating out the offshell heavy and light quarks and all the offshell gluons exactly reproduces the gauge invariant current in Eq. (45). 


\section{REFERENCES}

[1] C. W. Bauer, S. Fleming and M. Luke, Phys. Rev. D63, 014006 (2001).

[2] C. W. Bauer, S. Fleming, D. Pirjol and I. W. Stewart, Phys. Rev. D 63, 114020 (2001).

[3] C. W. Bauer and I. W. Stewart, Phys. Lett. B 516, 134 (2001).

[4] S. J. Brodsky and G. P. Lepage, in Perturbative Quantum Chromodynamics, Ed. by A. H. Mueller, World Scientific Publ., 1989, p. 93-240; G. P. Lepage and S. J. Brodsky, Phys. Rev. D 22, 2157 (1980).

[5] J.C. Collins, D.E. Soper, and G. Sterman in Perturbative Quantum Chromodynamics, Ed. by A. H. Mueller, World Scientific Publ., 1989, p. 1-93.

[6] M. Neubert, Phys. Rev. D 49, 4623 (1994); I. Bigi et al, Int. J. Mod. Phys. A 9, 2467 (1994).

[7] G. P. Korchemsky and G. Sterman, Phys. Lett. B 340, 96 (1994).

[8] G. P. Korchemsky and G. Marchesini, Nucl. Phys. B 406, 225 (1993).

[9] A. K. Leibovich and I. Z. Rothstein, Phys. Rev. D 61, 074006 (2000); A. K. Leibovich, I. Low and I. Z. Rothstein, Phys. Rev. D 62, 014010 (2000).

[10] S. J. Brodsky, hep-ph/9912340.

[11] S. J. Brodsky, hep-ph/0106294.

[12] H. D. Politzer and M. B. Wise, Phys. Lett. B 257, 399 (1991).

[13] M. Beneke, G. Buchalla, M. Neubert, and C. T. Sachrajda, Phys. Rev. Lett. 83, 1914 (1999).

[14] C. W. Bauer, D. Pirjol and I. W. Stewart, hep-ph/0107002.

[15] M. Beneke, V. A. Smirnov, Nucl. Phys. B 522, 321 (1998); V. A. Smirnov, Phys. Lett. B 404, 101 (1997); V. A. Smirnov, Theor. Math. Phys. 120, 870 (1999); V. A. Smirnov, Phys. Lett. B 465, 226 (1999).

[16] A. V. Manohar and M. B. Wise, Cambridge Monographs on Particle 68 Nuclear Physics, 8 Cosmology, Vol. 10.

[17] L. F. Abbott, Nucl. Phys. B 185, 189 (1981).

[18] R. Tucci, Phys. Rev. D 32, 945 (1985) [Erratum-ibid. D 34, 1235 (1985)].

[19] M. J. Dugan and B. Grinstein, Phys. Lett. B255, 583 (1991).

[20] P. Labelle, Phys. Rev. D 58, 093013 (1998); B. Grinstein and I.Z. Rothstein, Phys. Rev. D57, 78 (1998).

[21] See for example: J. C. Collins and D. E. Soper, Nucl. Phys. B 194, 445 (1982); J. C. Collins, D. E. Soper and G. Sterman, Nucl. Phys. B 261, 104 (1985); Nucl. Phys. B 308, 833 (1988).

[22] M. Beneke, G. Buchalla, M. Neubert, and C. T. Sachrajda, Nucl. Phys. B591, 313 (2000).

[23] G. P. Korchemsky and A. V. Radyushkin, Phys. Lett. B 279, 359 (1992).

[24] N. Isgur and M.B. Wise, Phys. Lett. B232 (1989) 113; Phys. Lett. B237 (1990) 527.

[25] P. P. Srivastava and S. J. Brodsky, Phys. Rev. D 61, 025013 (2000).

[26] J. B. Kogut and D. E. Soper, Phys. Lett. D1, 2901 (1970); J. D. Bjorken, J. B. Kogut and D. E. Soper, Phys. Rev. D3, 1382 (1971). 